Pacific

Journal of

Mathematics

COMPACT ANTI-DE SITTER 3-MANIFOLDS AND FOLDED HYPERBOLIC STRUCTURES ON SURFACES

FranÇOIS GuÉRITAUd, FANNY KASSEL AND MAXIME WOLFF 


\title{
COMPACT ANTI-DE SITTER 3-MANIFOLDS AND FOLDED HYPERBOLIC STRUCTURES ON SURFACES
}

\author{
François GuÉritaud, FANNy KaSSEl AND MaXime WolfF
}

\begin{abstract}
We prove that any non-Fuchsian representation $\rho$ of a surface group into $\operatorname{PSL}(2, \mathbb{R})$ is the holonomy of a folded hyperbolic structure on the surface, unless the image of $\rho$ is virtually abelian. Using this idea, we establish that any non-Fuchsian representation $\rho$ is strictly dominated by some Fuchsian representation $j$, in the sense that the hyperbolic translation lengths for $j$ are uniformly larger than for $\rho$. Conversely, any Fuchsian representation $j$ strictly dominates some non-Fuchsian representation $\rho$, whose Euler class can be prescribed. This has applications to the theory of compact anti-de Sitter 3-manifolds.
\end{abstract}

\section{Introduction}

Let $\Sigma_{g}$ be a closed, connected, oriented surface of genus $g$, with fundamental group $\Gamma_{g}=\pi_{1}\left(\Sigma_{g}\right)$, and let $\operatorname{Rep}_{g}^{\mathrm{fd}}$ and $\operatorname{Rep}_{g}^{\text {nfd }}$ be the sets of conjugacy classes of Fuchsian and non-Fuchsian representations of $\Gamma_{g}$ into $\operatorname{PSL}(2, \mathbb{R})$, respectively. The letters "fd" stand for "faithful, discrete". By work of Goldman [1988], the space $\operatorname{Hom}\left(\Gamma_{g}, \operatorname{PSL}(2, \mathbb{R})\right)$ of representations of $\Gamma_{g}$ into $\operatorname{PSL}(2, \mathbb{R})$ has $4 g-3$ connected components, indexed by the values of the Euler class

$$
\text { eu }: \operatorname{Hom}\left(\Gamma_{g}, \operatorname{PSL}(2, \mathbb{R})\right) \longrightarrow\{2-2 g, \ldots,-1,0,1, \ldots, 2 g-2\} .
$$

In the quotient, $\operatorname{Rep}_{g}^{\mathrm{fd}}$ consists of the two connected components of extremal Euler class, and $\operatorname{Rep}_{g}^{\text {nfd }}$ of all the other components of $\operatorname{Hom}\left(\Gamma_{g}, \operatorname{PSL}(2, \mathbb{R})\right) / \operatorname{PSL}(2, \mathbb{R})$.

1A. Strictly dominating representations. For any $g \in \operatorname{PSL}(2, \mathbb{R})$, let

$$
\lambda(g):=\inf _{p \in \mathbb{H}^{2}} d(p, g \cdot p) \geq 0
$$

be the translation length of $g$ in the hyperbolic plane $\mathbb{H}^{2}$. This defines a function

The authors were partially supported by the Agence Nationale de la Recherche under the grants DiscGroup (ANR-11-BS01-013), ETTT (ANR-09-BLAN-0116-01), ModGroup (ANR-11-BS010020), SGT (ANR-11-BS01-0018), and through the Labex CEMPI (ANR-11-LABX-0007-01). MSC2010: 20H10, 32G15, 53C50.

Keywords: representations of surface groups, folded hyperbolic structures, anti-de Sitter 3-manifolds. 
$\lambda: \operatorname{PSL}(2, \mathbb{R}) \rightarrow \mathbb{R}^{+}$which is invariant under conjugation. We say that an element $[j] \in \operatorname{Rep}_{g}^{\mathrm{fd}}$ strictly dominates an element $[\rho] \in \operatorname{Rep}_{g}^{\mathrm{nfd}}$ if

$$
\sup _{\gamma \in \Gamma_{g} \backslash\{1\}} \frac{\lambda(\rho(\gamma))}{\lambda(j(\gamma))}<1
$$

Note that (1-2) can never hold when $j$ and $\rho$ are both Fuchsian [Thurston 1986]. In this paper we prove the following:

Theorem 1.1. Any $[\rho] \in \operatorname{Rep}_{g}^{\mathrm{ndd}}$ is strictly dominated by some $[j] \in \operatorname{Rep}_{g}^{\mathrm{fd}}$. Any $[j] \in \operatorname{Rep}_{g}^{\mathrm{fd}}$ strictly dominates some $[\rho] \in \operatorname{Rep}_{g}^{\mathrm{nfd}}$, whose Euler class can be prescribed.

The first statement of Theorem 1.1 has been simultaneously and independently obtained by Deroin and Tholozan [2013] using more analytical methods. Their paper deals, more generally, with representations of $\Gamma_{g}$ into the isometry group of any complete, simply connected Riemannian manifold with sectional curvature at most -1 . They also announce a version for general CAT $(-1)$ spaces. The present methods, relying as they do on the Toponogov theorem (see Lemma 2.2 below), could likely extend to this general setting as well.

Our approach is constructive, using folded (or pleated) hyperbolic surfaces, as we now explain.

1B. Folded hyperbolic surfaces. Pleated hyperbolic surfaces were introduced by Thurston [1980] and play an important role in the theory of hyperbolic 3-manifolds. A folded hyperbolic surface is a pleated surface with all angles equal to 0 or $\pi$, whose holonomy takes values in $\operatorname{PSL}(2, \mathbb{R})$ (see Section $2 \mathrm{~B}$ ). It is easy to check (see [Thurston 1986, Proposition 2.1]) that the holonomy of a (nontrivially) folded hyperbolic structure on $\Sigma_{g}$ belongs to $\operatorname{Rep}_{g}^{\text {nfd }}$. In order to establish Theorem 1.1, we prove that the converse holds for representations whose image is not virtually abelian.

Theorem 1.2. An element of $\operatorname{Rep}_{g}^{\text {nfd }}$ is the holonomy of a folded hyperbolic structure on $\Sigma_{g}$ if and only if its image is not virtually abelian.

As usual, being virtually abelian means having an abelian subgroup of finite index. Besides abelian representations, Theorem 1.2 rules out dihedral representations, which preserve a geodesic line of $\mathbb{Q}^{2}$ and contain order-two symmetries of that line.

This result seems to have been known to experts since the work of Thurston [1980], but to our knowledge it is neither stated nor proved in the literature. Note that another type of folded hyperbolic structure was previously investigated by Goldman [1987].

We construct the folded hyperbolic structures of Theorem 1.2 explicitly, folding along geodesic laminations that are the union of simple closed curves and of maximal 
laminations of some pairs of pants (Proposition 3.1). More precisely, given a nonFuchsian representation $\rho$ whose image is not virtually abelian, we use a result of Gallo, Kapovich, and Marden [Gallo et al. 2000] to find a pants decomposition of $\Sigma_{g}$ such that the restriction of $\rho$ to any pair of pants $P$ is nonabelian and maps any cuff to a hyperbolic element. (The term cuff, always specific to a pair of pants, will in the sequel denote without distinction the homotopy class of a boundary component, or the geodesic in that class, or its length.) Folding along a certain maximal lamination in $P$ then gives a simple dictionary between the representations of the fundamental group of $P$ that have Euler class 0 and those that have Euler class \pm 1 (Lemma 3.6). The converse direction in Theorem 1.2 is elementary (Observation 2.7).

1C. Idea of the proof of Theorem 1.1. If $[\rho] \in \operatorname{Rep}_{g}^{\text {nfd }}$ is the holonomy of a folded hyperbolic structure on $\Sigma_{g}$, then the holonomy $\left[j_{0}\right] \in \operatorname{Rep}_{g}^{\mathrm{fd}}$ of the corresponding unfolded hyperbolic structure clearly dominates $[\rho]$ in the sense that $\lambda(\rho(\gamma)) \leq \lambda\left(j_{0}(\gamma)\right)$ for all $\gamma \in \Gamma_{g}$. In fact,

$$
\sup _{\gamma \in \Gamma_{g} \backslash\{1\}} \frac{\lambda(\rho(\gamma))}{\lambda\left(j_{0}(\gamma)\right)}=1,
$$

since any minimal component of the folding lamination can be approximated by simple closed curves. In order to prove Theorem 1.1 we need to make the domination strict.

To establish the first statement, the idea is, for $[\rho] \in \operatorname{Rep}_{g}^{\text {nfd }}$, to consider the holonomy $\left[j_{0}\right] \in \operatorname{Rep}_{g}^{\mathrm{fd}}$ of the unfolded hyperbolic structure given by Theorem 1.2, and to lengthen the closed curves (close to being) contained in the folding lamination while simultaneously not shortening the other curves too much. To do this, we work independently in each "folded subsurface" of $\Sigma_{g}$, which is a compact surface with boundary endowed with a hyperbolic structure induced by $j_{0}$. In each such subsurface we use a strip deformation construction due to Thurston [1986], which consists in adding hyperbolic strips to obtain a new hyperbolic metric with longer boundary components. We then glue back along the boundary components, after making sure that the lengths agree.

The second statement is easier in that it does not rely on Theorem 1.2. Starting with an element $[j] \in \operatorname{Rep}_{g}^{\mathrm{fd}}$, we choose a pants decomposition of $\Sigma_{g}$ along which to fold. To make sure that the cuffs of the pairs of pants will get contracted, we first deform $j$ slightly by negative strip deformations into another element $\left[j_{0}\right] \in \operatorname{Rep}_{g}^{\mathrm{fd}}$ with shorter cuffs, in such a way that the other curves do not get much longer. Folding $j_{0}$ then gives an element $[\rho] \in \operatorname{Rep}_{g}^{\text {nfd }}$ which is strictly dominated by $[j]$.

1D. An application to compact anti-de Sitter 3-manifolds. Theorem 1.1 has consequences for the theory of compact anti-de Sitter 3-manifolds. These are the 
compact Lorentzian 3-manifolds of constant negative curvature, i.e., the Lorentzian analogues of the compact hyperbolic 3-manifolds. They are locally modeled on the 3-dimensional anti-de Sitter space

$$
\operatorname{AdS}^{3}=\operatorname{PO}(2,2) / \mathrm{PO}(2,1),
$$

which is identified with $\operatorname{PSL}(2, \mathbb{R})$ endowed with the natural Lorentzian structure induced by the Killing form of its Lie algebra. The identity component of the isometry group of $\operatorname{AdS}^{3}$ is $\operatorname{PSL}(2, \mathbb{R}) \times \operatorname{PSL}(2, \mathbb{R})$, acting on $\operatorname{PSL}(2, \mathbb{R}) \simeq \operatorname{AdS}^{3}$ by right and left multiplication: $\left(g_{1}, g_{2}\right) \cdot g=g_{2} g g_{1}^{-1}$. All compact anti-de Sitter 3-manifolds are geodesically complete [Klingler 1996]. By [Kulkarni and Raymond 1985 ] and the Selberg lemma [1960, Lemma 8], they are quotients of PSL(2, $\mathbb{R})$ by torsion-free discrete subgroups $\Gamma$ of $\operatorname{PSL}(2, \mathbb{R}) \times \operatorname{PSL}(2, \mathbb{R})$ acting properly discontinuously, up to a finite covering; moreover, the groups $\Gamma$ are graphs of the form

$$
\Gamma=\left(\Gamma_{g}\right)^{j, \rho}:=\left\{(j(\gamma), \rho(\gamma)) \mid \gamma \in \Gamma_{g}\right\}
$$

for some $g \geq 2$, where $j, \rho \in \operatorname{Hom}\left(\Gamma_{g}, \operatorname{PSL}(2, \mathbb{R})\right)$ are representations and $j$ is Fuchsian, up to switching the two factors of $\operatorname{PSL}(2, \mathbb{R}) \times \operatorname{PSL}(2, \mathbb{R})$. In particular, $\Gamma \backslash \mathrm{AdS}^{3}$ is Seifert fibered over a hyperbolic base (see [Salein 1999, §3.4.2]).

Following [Salein 2000], we shall say that a pair $(j, \rho) \in \operatorname{Hom}\left(\Gamma_{g}, \operatorname{PSL}(2, \mathbb{R})\right)^{2}$ with $j$ Fuchsian is admissible if the action of $\left(\Gamma_{g}\right)^{j, \rho}$ on $\mathrm{AdS}^{3}$ is properly discontinuous. Note that $(j, \rho)$ is admissible if and only if its conjugates under $\operatorname{PSL}(2, \mathbb{R}) \times \operatorname{PSL}(2, \mathbb{R})$ are. Therefore, in order to understand the moduli space of compact anti-de Sitter 3-manifolds, we need to understand, for any $g \geq 2$, the space

$$
\operatorname{Adm}_{g} \subset \operatorname{Rep}_{g}^{\mathrm{fd}} \times \operatorname{Hom}\left(\Gamma_{g}, \operatorname{PSL}(2, \mathbb{R})\right) / \operatorname{PSL}(2, \mathbb{R})
$$

of conjugacy classes of admissible pairs $(j, \rho)$ with $j$ Fuchsian.

Examples of admissible pairs are readily obtained by taking $\rho$ to be trivial, or more generally with bounded image. The corresponding quotients of $\mathrm{AdS}^{3}$ are called standard. The first nonstandard examples were constructed by Goldman [1985] by deformation of standard ones - a technique later generalized by Kobayashi [1998]. Salein [2000] constructed the first examples of admissible pairs $(j, \rho)$ with $\mathrm{eu}(\rho) \neq 0$. He actually constructed examples where eu $(\rho)$ can take any nonextremal value. A necessary and sufficient condition for admissibility was given in [Kassel 2009]: a pair $(j, \rho)$ with $j$ Fuchsian is admissible if and only if $\rho$ is strictly dominated by $j$ in the sense of (1-2). In particular, by [Thurston 1986],

$$
\operatorname{Adm}_{g} \subset \operatorname{Rep}_{g}^{\mathrm{fd}} \times \operatorname{Rep}_{g}^{\mathrm{nfd}} .
$$

This properness criterion was extended in [Guéritaud and Kassel 2013] to quotients of $\mathrm{PO}(n, 1)=\operatorname{Isom}\left(\mathbb{H}^{n}\right)$ by discrete subgroups of $\mathrm{PO}(n, 1) \times \mathrm{PO}(n, 1)$ acting by left 
and right multiplication, for arbitrary $n \geq 2$ (recall that $\left.\operatorname{PSL}(2, \mathbb{R}) \simeq \operatorname{PO}(2,1)_{0}\right)$, and in [Guéritaud et al. 2015] to quotients of any simple Lie group $G$ of real rank 1 .

By completeness of compact anti-de Sitter manifolds [Klingler 1996], the Ehresmann-Thurston principle (see [Thurston 1980]) implies that $\mathrm{Adm}_{g}$ is open in $\operatorname{Rep}_{g}^{\mathrm{fd}} \times \operatorname{Rep}_{g}^{\mathrm{nfd}}$. Moreover, $\operatorname{Adm}_{g}$ has at least $4 g-5$ connected components, as Salein's examples show. Using the fact that the two connected components of $\operatorname{Rep}_{g}^{\mathrm{fd}}$ are conjugate under $\operatorname{PGL}(2, \mathbb{R})$, we can reformulate Theorem 1.1 as follows:

Corollary 1.3. The projections of $\mathrm{Adm}_{g}$ to $\operatorname{Rep}_{g}^{\mathrm{fd}}$ and to $\operatorname{Rep}_{g}^{\mathrm{nfd}}$ are both surjective. Moreover, for any connected components $\mathscr{C}_{1}$ of $\operatorname{Rep}{ }_{g}^{\mathrm{fd}}$ and $\mathscr{C}_{2}$ of $\operatorname{Rep} \mathrm{p}_{g}^{\mathrm{nfd}}$, the projections of $\operatorname{Adm}_{g} \cap\left(\mathscr{C}_{1} \times \mathscr{C}_{2}\right)$ to $\mathscr{C}_{1}$ and to $\mathscr{C}_{2}$ are both surjective.

The topology of $\mathrm{Adm}_{\mathrm{g}}$ is still unknown, but we believe that Corollary 1.3 (and the ideas behind its proof) could be used to prove that $\mathrm{Adm}_{g}$ is homeomorphic to $\operatorname{Rep}_{g}^{\mathrm{fd}} \times \operatorname{Rep}_{g}^{\mathrm{nfd}}$. Using the work of Hitchin [1987, Theorem 10.8 and Equation 10.6], this would give the homeomorphism type of the connected components of $\mathrm{Adm}_{g}$ corresponding to eu $(\rho) \neq 0$.

Furthermore, it would be interesting to obtain a geometric and combinatorial description of the fibers of the second projection $\operatorname{Adm}_{g} \rightarrow \operatorname{Rep}_{g}^{\text {nfd }}$. Such a description is given in [Danciger et al. 2014], in terms of the arc complex, in the different case that $j$ and $\rho$ are the holonomies of two convex cocompact hyperbolic structures on a given noncompact surface.

1E. Organization of the paper. In Section 2 we recall some facts about Lipschitz maps, folded hyperbolic structures, and the Euler class. Section 3 is devoted to the proof of Theorem 1.2, and Section 4 to that of Theorem 1.1.

\section{Reminders and useful facts}

2A. Lipschitz maps and their stretch locus. In the whole paper, we denote by $d$ the metric on the real hyperbolic plane $\mathbb{U}^{2}$. For a Lipschitz map $f: \mathbb{U}^{2} \rightarrow \mathbb{U}^{2}$ and a point $p \in \mathbb{H}^{2}$, we set:

- $\operatorname{Lip}(f):=\sup _{q \neq q^{\prime}} d\left(f(q), f\left(q^{\prime}\right)\right) / d\left(q, q^{\prime}\right) \geq 0$ (the Lipschitz constant);

- $\operatorname{Lip}_{p}(f):=\inf _{\varkappa} \operatorname{Lip}\left(\left.f\right|_{\ell}\right) \geq 0$, where $U$ ranges over all neighborhoods of $p$ in $\mathbb{\boxplus}^{2}$ (the local Lipschitz constant).

The function $p \mapsto \operatorname{Lip}_{p}(f)$ is upper semicontinuous:

$$
\operatorname{Lip}_{p}(f) \geq \limsup _{n \rightarrow+\infty} \operatorname{Lip}_{p_{n}}(f)
$$

for any sequence $\left(p_{n}\right)_{n \in \mathbb{N}}$ converging to $p$. The following is straightforward: 
Remark 2.1. For any rectifiable path $\mathscr{L} \subset \mathbb{M}^{2}$,

$$
\text { length }(f(\mathscr{L})) \leq \sup _{p \in \mathscr{L}} \operatorname{Lip}_{p}(f) \cdot \text { length }(\mathscr{L}) .
$$

In particular, if $\operatorname{Lip}_{p}(f) \leq C$ for all $p$ in a convex set $K$, then $\operatorname{Lip}\left(\left.f\right|_{K}\right) \leq C$.

2A1. The stretch locus. The following result is a particular case of [Guéritaud and Kassel 2013, Theorem 5.1]. It relies on the Toponogov theorem, a comparison theorem relating the curvature to the divergence rate of geodesics (see [Bridson and Haefliger 1999, Lemma II.1.13]).

Lemma 2.2 [Guéritaud and Kassel 2013]. Let $\Gamma$ be a torsion-free, finitely generated, discrete group and let $(j, \rho) \in \operatorname{Hom}(\Gamma, \operatorname{PSL}(2, \mathbb{R}))^{2}$ be a pair of representations with $j$ convex cocompact. Suppose the infimum of Lipschitz constants for all $(j, \rho)$-equivariant maps $f: \mathbb{T}^{2} \rightarrow \mathbb{M}^{2}$ is 1 , and the space $\mathscr{F}$ of maps achieving this infimum is nonempty. Then there exists a nonempty, $j(\Gamma)$-invariant geodesic lamination $\tilde{\Lambda}$ of $\mathbb{\boxplus}^{2}$ such that:

- any leaf of $\tilde{\Lambda}$ is isometrically preserved by all maps $f \in \mathscr{F}$;

- any connected component of $\mathbb{Q}^{2} \backslash \tilde{\Lambda}$ is either isometrically preserved by all $f \in \mathscr{F}$, or consists entirely of points $p$ at which $\operatorname{Lip}_{p}(f)<1$ for some $f \in \mathscr{F}_{F}$ (independent of $p$ ).

Definition 2.3. The union of $\tilde{\Lambda}$ and of the connected components of $\mathbb{H}^{2} \backslash \widetilde{\Lambda}$ that are isometrically preserved by all $f \in \mathscr{F}$ is called the stretch locus of $(j, \rho)$.

By convex cocompact we mean that $j$ is injective and discrete and that the group $j(\Gamma)$ does not contain any parabolic element. By $(j, \rho)$-equivariant we mean that $f(j(\gamma) \cdot p)=\rho(\gamma) \cdot f(p)$ for all $\gamma \in \Gamma$ and $p \in \mathbb{H}^{2}$. The space $\mathscr{F}$ is always nonempty, except possibly if $\rho(\Gamma)$ admits a unique fixed point in the boundary at infinity $\partial_{\infty} \mathbb{H}^{2}$ of $\mathbb{H}^{2}$ [Guéritaud and Kassel 2013, Lemma 4.11]. If $j$ and $\rho$ are conjugate under $\operatorname{PGL}(2, \mathbb{R})$, then the stretch locus of $(j, \rho)$ is the preimage of the convex core of $j(\Gamma) \backslash \mathbb{H}^{2}$. (This preimage is by definition the smallest nonempty $j(\Gamma)$-invariant closed convex subset of $\mathbb{M}^{2}$.)

2A2. Averaging Lipschitz maps. We now describe a technical tool for understanding the stretch locus. It is a procedure for averaging Lipschitz maps (see [Guéritaud and Kassel 2013, §2.5]), under which $\operatorname{Lip}_{p}$ behaves as it would for the barycenter of maps between affine Euclidean spaces. In Section 3D, we shall use this procedure with a partition of unity, as follows.

Let $\psi_{0}, \ldots, \psi_{n}: \mathbb{W}^{2} \rightarrow[0,1]$ be Lipschitz functions inducing a partition of unity on a subset $X$ of $\mathbb{H}^{2}$, subordinated to an open covering $B_{0} \cup \cdots \cup B_{n} \supset X$. For $0 \leq i \leq n$, let $\varphi_{i}: B_{i} \rightarrow \mathbb{M}^{2}$ be a Lipschitz map. For $p \in X$, let $I(p)$ be the collection 
of indices $i$ such that $p \in B_{i}$. Let $\sum_{i=0}^{n} \psi_{i} \varphi_{i}: X \rightarrow \mathbb{H}^{2}$ be the map sending any $p \in X$ to the minimizer in $\mathbb{\boxplus}^{2}$ of

$$
\sum_{i \in I(p)} \psi_{i}(p) d\left(\cdot, \varphi_{i}(p)\right)^{2}
$$

Then the following holds:

Lemma 2.4 [Guéritaud and Kassel 2013, Lemma 2.13]. The averaged map $\varphi:=$ $\sum_{i=0}^{n} \psi_{i} \varphi_{i}$ satisfies the "Leibniz rule"

$$
\operatorname{Lip}_{p}(\varphi) \leq \sum_{i \in I(p)}\left(\operatorname{Lip}_{p}\left(\psi_{i}\right) R(p)+\psi_{i}(p) \operatorname{Lip}_{p}\left(\varphi_{i}\right)\right)
$$

for all $p \in X$, where $R(p)$ is the diameter of the set $\left\{\varphi_{i}(p) \mid i \in I(p)\right\}$.

2A3. Admissibility. For any discrete group $\Gamma$ (not necessarily of the form $\Gamma_{g}$ ), we say that a pair of representations $(j, \rho) \in \operatorname{Hom}(\Gamma, \operatorname{PSL}(2, \mathbb{R}))^{2}$ is admissible if the group $\Gamma^{j, \rho}=\{(j(\gamma), \rho(\gamma)) \mid \gamma \in \Gamma\}$ acts properly discontinuously on $\operatorname{AdS}^{3}$. In this case, at least one of $j$ or $\rho$ is injective and discrete [Kassel 2008].

Understanding the stretch locus has led to the following necessary and sufficient conditions for admissibility. We denote by $\Gamma_{s}$ the set of nontrivial elements of $\Gamma$ corresponding to simple closed curves on the surface $j(\Gamma) \backslash \mathbb{U}^{2}$.

Theorem 2.5 [Kassel 2009; Guéritaud and Kassel 2013]. Let $\Gamma$ be a torsionfree, finitely generated, discrete group and let $j, \rho \in \operatorname{Hom}(\Gamma, \operatorname{PSL}(2, \mathbb{R}))$ be two representations with $j$ injective and discrete. The pair $(j, \rho)$ is admissible if and only if the following condition holds up to switching $j$ and $\rho$ :

(i) There exists a $(j, \rho)$-equivariant map $f: \mathbb{H}^{2} \rightarrow \mathbb{M}^{2}$ with $\operatorname{Lip}(f)<1$.

If $j$ is convex cocompact or if the group $\rho(\Gamma)$ does not have a unique fixed point in $\partial_{\infty} \mathbb{H}^{2}$, then (i) is equivalent to either of the following two conditions:

(ii) The representation $\rho$ is strictly dominated by $j$ :

$$
\sup _{\substack{\gamma \in \Gamma, \lambda(j(\gamma))>0}} \frac{\lambda(\rho(\gamma))}{\lambda(j(\gamma))}<1 ;
$$

(iii) The representation $\rho$ is strictly dominated by $j$ in restriction to simple closed curves:

$$
\sup _{\gamma \in \Gamma_{s}} \frac{\lambda(\rho(\gamma))}{\lambda(j(\gamma))}<1
$$

The implication (iii) $\Rightarrow$ (i) is nontrivial and relies on Lemma 2.2. The implications (i) $\Rightarrow$ (ii) $\Rightarrow$ (iii) are immediate modulo the following easy remark (see [Guéritaud and Kassel 2013, Lemma 4.5]): 
Remark 2.6. Let $\Gamma$ be a discrete group and $(j, \rho) \in \operatorname{Hom}(\Gamma, \operatorname{PSL}(2, \mathbb{R}))^{2}$ a pair of representations. For any $\gamma \in \Gamma$ and any $(j, \rho)$-equivariant Lipschitz map $f: \mathbb{H}^{2} \rightarrow \mathbb{M}^{2}$,

$$
\lambda(\rho(\gamma)) \leq \operatorname{Lip}(f) \lambda(j(\gamma)) .
$$

2B. Pleated and folded hyperbolic structures. Let $\Sigma$ be a connected, oriented surface of negative Euler characteristic, possibly with boundary, and let $\Gamma=\pi_{1}(\Sigma)$ be its fundamental group. Recall from [Bonahon 1996, §7] that a pleated hyperbolic structure on $\Sigma$ is a quadruple $(j, \rho, \Upsilon, f)$ where:

- $j \in \operatorname{Hom}(\Gamma, \operatorname{PSL}(2, \mathbb{R}))$ is the holonomy of a hyperbolic structure on $\Sigma$;

- $\rho \in \operatorname{Hom}(\Gamma, \operatorname{PSL}(2, \mathbb{C}))$ is a representation;

- $\Upsilon$ is a geodesic lamination on $\Sigma$;

- $f: \mathbb{H}^{2} \rightarrow \mathbb{H}^{3}$ is a $(j, \rho)$-equivariant, continuous map whose restriction to any connected component of $\mathbb{U}^{2} \backslash \widetilde{\Upsilon}$ is an isometric embedding. (Here we denote by $\tilde{\Upsilon}$ the preimage in $\mathbb{H}^{2}$ of $\Upsilon \subset \Sigma \simeq j(\Gamma) \backslash \mathbb{M}^{2}$.)

The representation $\rho$ is called the holonomy of the pleated hyperbolic structure. The closures of the connected components of $\mathbb{M}^{2} \backslash \widetilde{\Upsilon}$ are called the plates. Note that $f$ is 1-Lipschitz. For any $g, h \in \operatorname{PGL}(2, \mathbb{R})$,

$$
\left(g j(\cdot) g^{-1}, h \rho(\cdot) h^{-1}, \Upsilon, h \circ f \circ g^{-1}\right)
$$

is still a pleated hyperbolic structure on $\Sigma$.

Observation 2.7. Suppose that $\Sigma$ is compact. If $(j, \rho, \Upsilon, f)$ is a pleated hyperbolic structure on $\Sigma$, then the group $\rho(\Gamma)$ is not virtually abelian.

Proof. We see $\Sigma$ as the convex core of the hyperbolic surface $j(\Gamma) \backslash \mathbb{M}^{2}$. Consider a nondegenerate ideal triangle $T$ of $\mathbb{H}^{2}$ which is entirely contained in the intersection of one plate with the preimage of $\Sigma$ in $\mathbb{H}^{2}$. Let $\left(p_{n}\right)_{n \in \mathbb{N}}$ be a sequence of points of $T$ going to infinity. Since $\Sigma$ is compact, there exist $R>0$ and a sequence $\left(\gamma_{n}\right)_{n \in \mathbb{N}}$ of elements of $\Gamma$ such that $d\left(j\left(\gamma_{n}\right) \cdot p_{0}, p_{n}\right) \leq R$ for all $n \in \mathbb{N}$. Since $f$ is $(j, \rho)$-equivariant and 1-Lipschitz,

$$
d\left(\rho\left(\gamma_{n}\right) \cdot f\left(p_{0}\right), f\left(p_{n}\right)\right) \leq d\left(j\left(\gamma_{n}\right) \cdot p_{0}, p_{n}\right) \leq R
$$

for all $n \in \mathbb{N}$. Applying this to sequences $\left(p_{n}\right)$ converging to the three ideal vertices of $T$, and using the fact that the restriction of $f$ to $T$ is an isometry, we see that the limit set of $\rho(\Gamma)$ contains at least three points. In particular, $\rho(\Gamma)$ is not virtually abelian.

We shall also use the following elementary remark: 
Remark 2.8. Let $(j, \rho, \Upsilon, f)$ be a pleated hyperbolic structure on $\Sigma$. If some leaf of $\Upsilon$ spirals to a boundary component of $\Sigma$ corresponding to an element $\gamma \in \Gamma$, then $\lambda(j(\gamma))=\lambda(\rho(\gamma))$, where $\lambda: \operatorname{PSL}(2, \mathbb{C}) \rightarrow \mathbb{R}^{+}$is the translation length function in $\mathbb{H}^{3}$ extending (1-1).

Any pleated hyperbolic structure $(j, \rho, \Upsilon, f)$ on $\Sigma$ defines a bending cocycle, i.e., a map $\beta$ from the set of pairs of plates to $\mathbb{R} / 2 \pi \mathbb{Z}$ which is symmetric and additive:

$$
\beta(P, Q)=\beta(Q, P) \quad \text { and } \quad \beta(P, Q)+\beta(Q, R)=\beta(P, R)
$$

for all plates $P, Q, R$. Intuitively, $\beta(P, Q)$ is the total angle of pleating encountered when traveling from $f(P)$ to $f(Q)$ along $f\left(\mathbb{M}^{2}\right)$ in $\mathbb{H}^{3}$. Conversely, to any bending cocycle, Bonahon [1996, §8] associates a pleated surface.

In this paper we consider a special case of pleated surfaces $(j, \rho, \Upsilon, f)$, namely those for which $f$ takes values in a copy of $\mathbb{U}^{2}$ inside $\mathbb{U}^{3}$ (i.e., in a totally geodesic plane) and $\rho$ takes values in $\operatorname{Isom}^{+}\left(\mathbb{H}^{2}\right)=\operatorname{PSL}(2, \mathbb{R})$. In this case, we speak of a folded hyperbolic structure on $\Sigma$, and say that $\rho$ is a folding of $j$. The map $f$ defines a coloring of $\Sigma \backslash \Upsilon$, i.e., a $j(\Gamma)$-invariant function $\tilde{c}$ from the set of plates to $\{-1,1\}$. Namely, we set $\tilde{c}(P)=-1$ if the restriction of $f$ to $P$ is orientationpreserving, and $\tilde{c}(P)=1$ otherwise. Note that the bending cocycle of a folded hyperbolic structure is valued in $\{0, \pi\}$ : for all plates $P$ and $Q$,

$$
\beta(P, Q)=\frac{1}{2}(1-\tilde{c}(P) \tilde{c}(Q)) \pi \in\{0, \pi\} .
$$

The coloring $\tilde{c}$ descends to a continuous, locally constant function $c$ from $\Sigma \backslash \Upsilon$ to $\{-1,1\}$. Conversely, any such function, after lifting to a coloring $\tilde{c}$ from the set of connected components of $\mathbb{U}^{2} \backslash \tilde{\Upsilon}$ to $\{-1,1\}$, defines a bending cocycle on $\mathbb{U}^{2} \backslash \tilde{\Upsilon}$ by the formula (2-1). This bending cocycle, in turn, defines a folded hyperbolic structure on $\Sigma$, by the work of Bonahon [1996].

2C. The Euler class. We now give a brief introduction to the Euler class, along the lines of [Wolff 2011, §2.3.3]. For details and complements we refer to [Ghys 2001] or [Calegari 2004, §2].

As in the introduction, let $\Sigma_{g}$ be a closed, connected, oriented surface of genus $g \geq 2$ with fundamental group $\Gamma_{g}$. The Euler class of a representation $\rho \in \operatorname{Hom}\left(\Gamma_{g}, \operatorname{PSL}(2, \mathbb{R})\right)$ measures the obstruction to lifting $\rho$ to the universal cover $\widetilde{P S L}(2, \mathbb{R})$ of PSL $(2, \mathbb{R})$, and its parity measures the obstruction to lifting $\rho$ to $\operatorname{SL}(2, \mathbb{R})$. To define the Euler class, choose a set-theoretic section $s$ of the covering map $\widetilde{\operatorname{PSL}}(2, \mathbb{R}) \rightarrow \operatorname{PSL}(2, \mathbb{R})$. Consider a triangulation of $\Sigma_{g}$ with a vertex at the basepoint $x_{0}$ defining $\Gamma_{g}=\pi_{1}\left(\Sigma_{g}, x_{0}\right)$, and choose an orientation on every edge of the triangulation. Choose a maximal tree in the 1-skeleton of the triangulation and, for every oriented edge $\sigma$ in this tree, set $\rho(\sigma):=1 \in \operatorname{PSL}(2, \mathbb{R})$. Any other oriented 
edge $\sigma^{\prime}$ corresponds (by closing up in the unique possible way along the rooted tree) to an element $\gamma \in \Gamma_{g}$, and we set $\rho\left(\sigma^{\prime}\right):=\rho(\gamma) \in \operatorname{PSL}(2, \mathbb{R})$. The boundary of any oriented triangle $\tau$ of the triangulation can be written as $\sigma_{1}^{\varepsilon_{1}} \sigma_{2}^{\varepsilon_{2}} \sigma_{3}^{\varepsilon_{3}}$, where $\sigma_{1}, \sigma_{2}, \sigma_{3}$ are edges with the chosen orientation and $\varepsilon_{i} \in\{ \pm 1\}$. We set

$$
\mathrm{eu}(\rho)(\tau):=s\left(\rho\left(\sigma_{1}\right)\right)^{\varepsilon_{1}} s\left(\rho\left(\sigma_{2}\right)\right)^{\varepsilon_{2}} s\left(\rho\left(\sigma_{3}\right)\right)^{\varepsilon_{3}} .
$$

Summing over triangles $\tau$, this defines an element of $H^{2}\left(\Sigma_{g}, \pi_{1}(\operatorname{PSL}(2, \mathbb{R}))\right)$, hence an element of $H^{2}\left(\Sigma_{g}, \mathbb{Z}\right)$ under the identification $\pi_{1}(\operatorname{PSL}(2, \mathbb{R})) \simeq \mathbb{Z}$. This element eu $(\rho) \in H^{2}\left(\Sigma_{g}, \mathbb{Z}\right)$ is called the Euler class of $\rho$. Its evaluation on the fundamental class in $H_{2}\left(\Sigma_{g}, \mathbb{Z}\right)$ is an integer, which we still call the Euler class of $\rho$. It is invariant under conjugation by $\operatorname{PSL}(2, \mathbb{R})$, and changes sign under conjugation by $\operatorname{PGL}(2, \mathbb{R}) \backslash \operatorname{PSL}(2, \mathbb{R})$.

We can also define the Euler class for representations of the fundamental group of a compact, connected, oriented surface $\Sigma$ with boundary, of negative Euler characteristic, provided that the boundary curves are sent to hyperbolic elements. Indeed, any hyperbolic element $g \in \operatorname{PSL}(2, \mathbb{R})$ has a canonical lift to $\widetilde{\operatorname{PSL}}(2, \mathbb{R})$ because it belongs to a unique one-parameter subgroup of $\operatorname{PSL}(2, \mathbb{R})$, which defines a path from the identity to $g$. Choose a section $s$ of the projection $\widetilde{\operatorname{PSL}}(2, \mathbb{R}) \rightarrow \operatorname{PSL}(2, \mathbb{R})$ that maps any hyperbolic element to its canonical lift. Then the construction above, using triangulations of $\Sigma$ containing exactly one vertex on each boundary component, defines an Euler class, independent of all choices.

For instance, let $\Sigma$ be an oriented pair of pants with fundamental group $\Gamma=$ $\langle\alpha, \beta, \gamma \mid \alpha \beta \gamma=1\rangle$, where $\alpha, \beta, \gamma$ correspond to the three boundary curves, endowed with the orientation induced by the surface. For any representation $\rho \in \operatorname{Hom}(\Gamma, \operatorname{PSL}(2, \mathbb{R}))$ with $\rho(\alpha), \rho(\beta), \rho(\gamma)$ hyperbolic,

$$
\mathrm{eu}(\rho)=s(\rho(\alpha)) s(\rho(\beta)) s(\rho(\gamma)) \in Z(\widetilde{\mathrm{PSL}}(2, \mathbb{R})) \simeq \mathbb{Z}
$$

In particular, eu $(\rho) \in\{-1,0,1\}$, and $|\mathrm{eu}(\rho)|=1$ if and only if $\rho$ is the holonomy of a hyperbolic structure on $\Sigma$, possibly after reversing the orientation. If $s^{\prime}$ is a section of the projection $\operatorname{SL}(2, \mathbb{R}) \rightarrow \operatorname{PSL}(2, \mathbb{R})$ that maps any hyperbolic element to its lift of positive trace, then (2-2) implies

$$
s^{\prime}(\rho(\alpha)) s^{\prime}(\rho(\beta)) s^{\prime}(\rho(\gamma))=(-\mathrm{Id})^{\mathrm{eu}(\rho)} .
$$

By construction, the Euler class is additive: if $\Sigma$ is the union of two subsurfaces $\Sigma^{\prime}$ and $\Sigma^{\prime \prime}$ glued along curves $\gamma_{i}$, and if $\rho \in \operatorname{Hom}\left(\pi_{1}(\Sigma), \operatorname{PSL}(2, \mathbb{R})\right)$ is a representation sending all the curves $\gamma_{i}$ (and the boundary curves of $\Sigma$, if any) to hyperbolic elements of $\operatorname{PSL}(2, \mathbb{R})$, then eu $(\rho)$ is the sum of the Euler classes of the restrictions of $\rho$ to the fundamental groups of $\Sigma^{\prime}$ and $\Sigma^{\prime \prime}$. This implies that a folded hyperbolic structure defined by a coloring $c$ from the set $\mathscr{P}$ of connected components of 

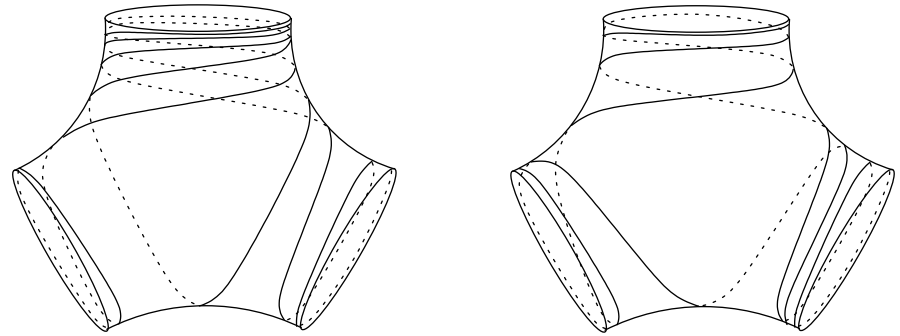

Figure 1. A pair of pants carries 24 maximal geodesic laminations containing a geodesic spiraling from a boundary component to itself (left), and 8 triskelion laminations (right).

$\Sigma \backslash \Upsilon$ to $\{-1,1\}$ has Euler class $\frac{1}{2 \pi} \sum_{P \in \mathscr{P}} c(P) \mathscr{A}(P)$, where $\mathscr{A}(P)$ is the area of $P$.

We shall use the following terminology:

Definition 2.9. A representation $\rho \in \operatorname{Hom}\left(\pi_{1}(\Sigma), \operatorname{PSL}(2, \mathbb{R})\right)$ is geometric if it maps the boundary curves of $\Sigma$ to hyperbolic elements of PSL $(2, \mathbb{R})$ and has extremal Euler class or, equivalently, if it is the holonomy of a hyperbolic structure on $\Sigma$, possibly after reversing the orientation.

2D. Laminations in a pair of pants. A hyperbolic pair of pants $\Sigma$ carries only finitely many geodesic laminations, because only 21 geodesics are simple — namely 3 closed geodesics (the boundary components), 6 geodesics spiraling from a boundary component to itself, and 12 geodesics spiraling from a boundary component to another. It admits 32 ideal triangulations, of which 24 contain a geodesic spiraling from a boundary component to itself and the other 8 do not (see Figure 1). We shall call the laminations corresponding to these 8 triangulations the triskelion laminations of $\Sigma$. They differ by the spiraling directions of the spikes of the triangles at each boundary component.

\section{Holonomies of folded hyperbolic structures}

Let $\lambda: \operatorname{PSL}(2, \mathbb{R}) \rightarrow \mathbb{R}^{+}$be the translation length function (1-1). For any representation $\rho \in \operatorname{Hom}\left(\Gamma_{g}, \operatorname{PSL}(2, \mathbb{R})\right)$, we set

$$
\lambda_{\rho}:=\lambda \circ \rho: \Gamma_{g} \longrightarrow \mathbb{R}^{+} .
$$

The function $\lambda_{\rho}$ is identically zero if and only if the group $\rho\left(\Gamma_{g}\right)$ is unipotent or bounded. The goal of this section is to prove the following:

Proposition 3.1. For any $[\rho] \in \operatorname{Rep}_{g}^{\mathrm{nfd}}$ with $\lambda_{\rho} \not \equiv 0$, there exist elements $\left[j_{0}\right],\left[j_{0}^{\prime}\right]$ of $\operatorname{Rep}_{g}^{\mathrm{fd}}$ and a decomposition $\Pi$ of $\Sigma_{g}$ into pairs of pants, each labeled $-1,0$, or 1 , with the following properties: 
(1) For any representations $j_{0}, \rho$ in the respective classes $\left[j_{0}\right],[\rho]$, there is a 1-Lipschitz, $\left(j_{0}, \rho\right)$-equivariant map $f: \mathbb{T}^{2} \rightarrow \mathbb{T}^{2}$ that is an orientationpreserving (resp. orientation-reversing) isometry in restriction to any connected subset of $\mathbb{M}^{2}$ projecting to a union of pants labeled -1 (resp. 1) in $j_{0}\left(\Gamma_{g}\right) \backslash \mathbb{M}^{2} \simeq$ $\Sigma_{g}$, and that satisfies $\operatorname{Lip}_{p}(f)<1$ for any $p \in \mathbb{H}^{2}$ projecting to the interior of a pair of pants labeled 0 ;

(2) For any representations $j_{0}^{\prime}, \rho$ in the respective classes $\left[j_{0}^{\prime}\right],[\rho]$, if the group $\rho\left(\Gamma_{g}\right)$ is not virtually abelian, then $\rho$ is a folding of $j_{0}^{\prime}$ along a lamination $\Upsilon$ of $\Sigma_{g}$ consisting of all the cuffs together with a triskelion lamination inside each pair of pants labeled 0 , with the coloring $c: \Sigma_{g} \backslash \Upsilon \rightarrow\{-1,1\}$ taking the value -1 (resp. 1) on each pair of pants labeled -1 (resp. 1), and both values on each pair of pants labeled 0 ;

(3) $\left[j_{0}\right]$ and $\left[j_{0}^{\prime}\right]$ only differ by earthquakes along the cuffs of the pairs of pants of the decomposition.

Property (1) is used to prove Theorem 1.1 in Section 4, while (2) is a more precise statement of Theorem 1.2. We refer to Section 2A for the notation $\operatorname{Lip}_{p}(f)$ and to Section 2D for triskelion laminations. By additivity (see Section 2C), the Euler class of $\rho$ is the sum of the labels of the pairs of pants.

Proposition 3.1 is proved by choosing an appropriate pants decomposition (Section 3A) and understanding the representations of the fundamental group of a pair of pants (Section 3B). These ingredients are brought together in Section 3C. In Section 3D we present a variation on Proposition 3.1(1), which is later used to prove the second statement of Theorem 1.1.

\section{A. Pants decompositions. Our first ingredient is the following:}

Lemma 3.2. For any $[\rho] \in \operatorname{Rep}_{g}^{\mathrm{nfd}}$ with $\lambda_{\rho} \not \equiv 0$, there is a pants decomposition of $\Sigma_{g}$ such that $\rho$ maps any cuff to a hyperbolic element. If $\rho\left(\Gamma_{g}\right)$ is not virtually abelian, then we may assume that the restriction of $\rho$ to the fundamental group of any pair of pants of the decomposition is nonabelian.

Recall that $[\rho] \in \operatorname{Rep}_{g}^{\text {nfd }}$ is said to be elementary if the group $\rho\left(\Gamma_{g}\right)$ admits a finite orbit in $\mathbb{U}^{2}$ or in $\partial_{\infty} \mathbb{H}^{2}$. In the case that $[\rho]$ is not elementary, Lemma 3.2 is contained in the following result of Gallo, Kapovich, and Marden:

Lemma 3.3 [Gallo et al. 2000, part A]. For any nonelementary $[\rho] \in \operatorname{Rep}_{g}^{\text {nfd }}$, there is a pants decomposition of $\Sigma_{g}$ such that the fundamental group of any pair of pants maps injectively to a 2-generator Schottky group under $\rho$.

We now treat the case that $\rho$ is elementary.

Proof of Lemma 3.2 when $\rho$ is elementary. By induction, Lemma 3.2 is a consequence of the following two claims: 
Claim 3.4. Let $\Sigma$ be a connected compact surface of genus $g \geq 1$ with $k \geq 0$ boundary components such that $\chi(\Sigma)=2-2 g-k<0$, and let $\rho \in \operatorname{Hom}\left(\pi_{1}(\Sigma), \operatorname{PSL}(2, \mathbb{R})\right)$ be an elementary representation with $\lambda_{\rho} \not \equiv 0$ sending each boundary curve of $\Sigma$ (if any) to a hyperbolic element. Then we can cut $\Sigma$ open along some nonseparating simple closed curve whose image under $\rho$ is a hyperbolic element, yielding a new surface $\Sigma^{\prime}$ of genus $g-1$ and an induced representation $\rho^{\prime} \in \operatorname{Hom}\left(\pi_{1}\left(\Sigma^{\prime}\right), \operatorname{PSL}(2, \mathbb{R})\right)$ sending all $k+2$ boundary curves of $\Sigma^{\prime}$ to hyperbolic elements. If the image of $\rho$ is not virtually abelian, then the image of $\rho^{\prime}$ is not virtually abelian.

Claim 3.5. Let $\Sigma$ be a connected compact surface of genus $g=0$ with $k \geq 4$ boundary components, and let $\rho \in \operatorname{Hom}\left(\pi_{1}(\Sigma), \operatorname{PSL}(2, \mathbb{R})\right)$ be an elementary representation sending each boundary curve of $\Sigma$ to a hyperbolic element. Then we can cut $\Sigma$ along some simple closed curve of $\Sigma$, not freely homotopic to a boundary component, whose image under $\rho$ is a hyperbolic element, yielding two new surfaces $\Sigma_{1}$ and $\Sigma_{2}$ with lower complexity and two induced representations $\rho_{i} \in \operatorname{Hom}\left(\pi_{1}\left(\Sigma_{i}\right), \operatorname{PSL}(2, \mathbb{R})\right)$ sending each boundary curve to a hyperbolic element. If the image of $\rho$ is nonabelian, then we can do this in such a way that the images of the $\rho_{i}$ are nonabelian.

Proof of Claim 3.4. We first observe that $\pi_{1}(\Sigma)$ is generated by elements representing nonseparating simple closed curves on $\Sigma$. Indeed, consider a standard presentation

$$
\pi_{1}(\Sigma)=\left\langle a_{1}, b_{1}, \ldots, a_{g}, b_{g}, c_{1}, \ldots, c_{k} \mid\left[a_{1}, b_{1}\right] \cdots\left[a_{g}, b_{g}\right] c_{1} \cdots c_{k}=1\right\rangle
$$

of $\pi_{1}(\Sigma)$ by generators and relations, where $a_{i}, b_{i}$ represent nonseparating simple closed curves and $c_{i}$ a curve freely homotopic to a boundary component. Either $a_{1} c_{i}$ represents a nonseparating simple closed curve for all $i$, or $a_{1}^{-1} c_{i}$ represents a nonseparating simple closed curve for all $i$. Thus we may take the generating set $\left\{a_{1}, b_{1}, \ldots, a_{g}, b_{g}, a_{1}^{\varepsilon} c_{1}, \ldots, a_{1}^{\varepsilon} c_{k}\right\}$ for some $\varepsilon \in\{-1,1\}$.

Let us show that $\rho$ sends some nonseparating simple closed curve of $\Sigma$ to a hyperbolic element. Since $\lambda_{\rho} \not \equiv 0$, two mutually exclusive situations are possible:

(T) The group $\rho\left(\pi_{1}(\Sigma)\right)$ has a fixed point $\xi$ in $\partial_{\infty} \mathbb{H}^{2}$; it is then conjugate to a group of triangular (possibly diagonal) matrices in $\operatorname{PSL}(2, \mathbb{R})$.

(VA) The group $\rho\left(\pi_{1}(\Sigma)\right)$ preserves a geodesic line $\ell$ of $\mathbb{H}^{2}$ and contains both translations along $\ell$ and order-two symmetries of $\ell$ reversing its orientation; it is then virtually abelian but not abelian.

Consider a system $F$ of generators of $\pi_{1}(\Sigma)$ representing nonseparating simple closed curves. In case (T), some element of $F$ is necessarily sent by $\rho$ to a hyperbolic element: otherwise the group $\rho\left(\pi_{1}(\Sigma)\right)$ would contain only parabolic elements and the identity, which would contradict the fact that $\lambda_{\rho} \not \equiv 0$. Suppose we are in 
case (VA) and $\rho$ does not send any element of $F$ to a hyperbolic element; it then sends some element $\gamma \in F$ to an order-two symmetry of $\ell$ (because it is not the constant homomorphism). We may complete $\gamma$ into a new standard presentation of the form (3-1) with $\gamma=a_{1}$. Consider the generating set

$$
F^{\prime}=\left\{b_{1}, a_{1} b_{1}, a_{2}^{-1} b_{1}, b_{2} b_{1}, \ldots, a_{g}^{-1} b_{1}, b_{g} b_{1}, c_{1}^{\varepsilon} b_{1}, \ldots, c_{k}^{\varepsilon} b_{1}\right\},
$$

where $\varepsilon \in\{-1,1\}$. If $\varepsilon$ is suitably chosen, then every $\gamma^{\prime} \in F^{\prime}$ represents a nonseparating simple closed curve, and $\gamma^{\prime}$ and $\gamma=a_{1}$ are standard generators of a one-holed torus embedded in $\Sigma$; it follows that $\gamma \gamma^{\prime}$ represents a nonseparating simple closed curve as well. Necessarily, there exists $\gamma^{\prime} \in F^{\prime}$ such that $\rho\left(\gamma^{\prime}\right)$ does not commute with $\rho(\gamma)$ : otherwise the group $\rho\left(\pi_{1}(\Sigma)\right)$ would be contained in the centralizer of $\rho(\gamma)$, which is compact, and this would contradict the fact that $\lambda_{\rho} \not \equiv 0$. Either this $\rho\left(\gamma^{\prime}\right)$ is hyperbolic, or it is an order-two symmetry whose center is different from that of $\rho(\gamma)$, in which case $\rho\left(\gamma \gamma^{\prime}\right)$ is hyperbolic. In either case we have found a nonseparating simple closed curve whose image in $\pi_{1}(\Sigma)$ is mapped by $\rho$ to a hyperbolic element.

Let $\Sigma^{\prime}$ be obtained by cutting $\Sigma$ open along such a simple closed curve. If the image of the induced representation $\rho^{\prime} \in \operatorname{Hom}\left(\pi_{1}\left(\Sigma^{\prime}\right), \operatorname{PSL}(2, \mathbb{R})\right)$ is virtually abelian, then so is the image of $\rho$. Indeed, $\pi_{1}(\Sigma)$ is generated by $\pi_{1}\left(\Sigma^{\prime}\right)$ together with an element $\gamma^{\prime}$ that conjugates two elements of $\pi_{1}\left(\Sigma^{\prime}\right)$ with hyperbolic images under $\rho^{\prime}$. If the image of $\rho^{\prime}$ is virtually abelian, preserving some geodesic line $\ell$ of $\mathbb{\boxplus}^{2}$, then $\rho\left(\gamma^{\prime}\right)$ has to preserve $\ell$, and so does the whole image of $\rho$. Thus the image of $\rho$ is virtually abelian.

Proof of Claim 3.5. Since the boundary curves of $\Sigma$ generate $\pi_{1}(\Sigma)$ and since they all have hyperbolic image under the elementary representation $\rho$, the group $\rho\left(\pi_{1}(\Sigma)\right)$ has a fixed point $\xi$ in $\partial_{\infty} \mathbb{H}^{2}$ (case (T) above). Choose a geodesic line $\ell$ of $\mathbb{U}^{2}$ with endpoint $\xi$. For any $\gamma \in \Gamma$ we may write in a unique way $\rho(\gamma)=a_{\gamma} u_{\gamma}$, where $a_{\gamma}$ belongs to the stabilizer $A$ of $\xi$ and $\ell$ in $\operatorname{PSL}(2, \mathbb{R})$, and $u_{\gamma} \in \operatorname{PSL}(2, \mathbb{R})$ is unipotent or trivial. The map $\gamma \mapsto a_{\gamma}$ can be seen as a nonzero element $\omega$ of $H^{1}\left(\Sigma_{g}, \mathbb{R}\right)$ after identifying $A$ with $(\mathbb{R},+)$. Consider a standard presentation

$$
\pi_{1}(\Sigma)=\left\langle c_{1}, \ldots, c_{k} \mid c_{1} \cdots c_{k}=1\right\rangle
$$

of $\pi_{1}(\Sigma)$ by generators and relations, where $c_{1}, \ldots, c_{k}$ represent curves freely homotopic to the boundary components of $\Sigma$, and $c_{i} c_{j}$ represents a simple curve for any $i<j$. We claim that $\rho$ sends one of the $c_{i} c_{j}$ to a hyperbolic element. Indeed, otherwise we would have $\omega\left(c_{i}\right)+\omega\left(c_{j}\right)=0$ for all $i \neq j$; solving this linear system gives $\omega\left(c_{i}\right)=0$ for all $i$, which would contradict the assumption that $\rho\left(c_{i}\right)$ is hyperbolic.

For $1 \leq i \leq k$, let $\xi_{i} \in \partial_{\infty} \mathbb{H}^{2}$ be the fixed point of $\rho\left(c_{i}\right)$ that is different from $\xi$. If the image of $\rho$ is not abelian, then there exists $i$ such that $\xi_{i} \neq \xi_{i+1}$ (with the 
convention that $\xi_{k+1}=\xi_{1}$ ). Precomposing $\rho$ by a Dehn twist along a curve freely homotopic to $c_{i} c_{i+1}$ corresponds to conjugating $\rho\left(c_{i}\right)$ and $\rho\left(c_{i+1}\right)$ by $\rho\left(c_{i} c_{i+1}\right)$ while leaving all the other $\rho\left(c_{j}\right)$ unchanged. Applying a large enough power of this Dehn twist, with the appropriate sign if $\rho\left(c_{i} c_{i+1}\right)$ is hyperbolic, pushes $\xi_{i}$ and $\xi_{i+1}$ to two distinct points arbitrarily close to $\xi$; in particular, we can make $\xi_{i}$ and $\xi_{i+1}$ distinct from the other points $\xi_{j}$. We then proceed similarly with the new point $\xi_{i+1}$ and $\xi_{i+2}$, and so on, until all the points $\xi_{i}$ are pairwise distinct. We then conclude as above: one of the $c_{i} c_{j}$ (with $i \neq j$ ) has hyperbolic image under $\rho$. It represents a curve cutting $\Sigma$ into two smaller surfaces on which $\rho$ induces nonabelian representations.

To prove Lemma 3.2, just make repeated use of Claim 3.4 to reduce to a surface of genus 0 , then of Claim 3.5 to decompose it into pairs of pants.

3B. Representations of the fundamental group of a pair of pants. The following lemma gives a dictionary between the geometric and nongeometric representations (Definition 2.9) of the fundamental group of a pair of pants.

Lemma 3.6. Let $\Gamma=\langle\alpha, \beta, \gamma \mid \alpha \beta \gamma=1\rangle$ be the fundamental group of a pair of pants $\Sigma$, with $\alpha, \beta, \gamma$ corresponding to the three boundary curves.

For any $a, b, c>0$ such that none is the sum of the other two, there are exactly two representations $\tau \in \operatorname{Hom}(\Gamma, \operatorname{PSL}(2, \mathbb{R}))$ satisfying

$$
\left(\lambda_{\tau}(\alpha), \lambda_{\tau}(\beta), \lambda_{\tau}(\gamma)\right)=(a, b, c)
$$

up to conjugation under $\operatorname{PGL}(2, \mathbb{R})$. One of them is geometric (with $|\mathrm{eu}(\tau)|=1$ ). The other is nongeometric (with $\mathrm{eu}(\tau)=0$ ), and is obtained from the geometric one by folding along any of the eight triskelion laminations of $\Sigma$.

For any $a, b, c>0$ such that one is the sum of the other two, there are exactly four representations $\tau \in \operatorname{Hom}(\Gamma, \operatorname{PSL}(2, \mathbb{R}))$ satisfying (3-2), up to conjugation under $\operatorname{PGL}(2, \mathbb{R})$. One of them is geometric (with $|\mathrm{eu}(\tau)|=1$ ). The other three are elementary (with $\mathrm{eu}(\tau)=0$ ): two have an image that is not virtually abelian and the third one is their abelianization. Each of the two nonabelian elementary representations is obtained from the geometric one by folding along any of four different triskelion laminations of $\Sigma$.

When one of $a, b, c$ is the sum of the other two, the images of the two nonabelian elementary representations $\tau \in \operatorname{Hom}(\Gamma, \operatorname{PSL}(2, \mathbb{R}))$ are conjugate to triangular matrices; their abelianization is by definition their projection to the group of diagonal matrices.

Proof. Fix $a, b, c>0$. We first determine the number of conjugacy classes of representations $\tau$ satisfying (3-2). Set $(A, B, C):=\left(e^{a / 2}, e^{b / 2}, e^{c / 2}\right)$, and let 
$\tau \in \operatorname{Hom}(\Gamma, \operatorname{PSL}(2, \mathbb{R}))$ satisfy (3-2). Up to conjugating $\tau$ by $\operatorname{PGL}(2, \mathbb{R})$, we can find lifts $\bar{\tau}(\alpha) \in \operatorname{SL}(2, \mathbb{R})$ of $\tau(\alpha)$ and $\bar{\tau}(\beta) \in \operatorname{SL}(2, \mathbb{R})$ of $\tau(\beta)$ of the form

$$
\bar{\tau}(\alpha)=\left(\begin{array}{ll}
A & 0 \\
0 & A^{-1}
\end{array}\right) \text { and } \bar{\tau}(\beta)=\left(\begin{array}{cc}
B+x & y \\
z & B^{-1}-x
\end{array}\right)
$$

with $x, y, z \in \mathbb{R}$. Since $\alpha$ and $\beta$ freely generate $\Gamma$, this determines a lift $\bar{\tau}$ of $\tau$ in $\operatorname{Hom}(\Gamma, \operatorname{SL}(2, \mathbb{R}))$. The sign $\varepsilon \in\{ \pm 1\}$ of $\operatorname{Tr}(\bar{\tau}(\alpha)) \operatorname{Tr}(\bar{\tau}(\beta)) \operatorname{Tr}(\bar{\tau}(\gamma))$ does not depend on the choice of $\bar{\tau}(\alpha)$ and $\bar{\tau}(\beta)$. By (2-2), we have eu $(\tau) \in\{-1,0,1\}$, with $|\mathrm{eu}(\tau)|=1$ if and only if $\tau$ is geometric, and by (2-3)

$$
\varepsilon=(-1)^{\mathrm{eu}(\tau)} .
$$

The trace of $\bar{\tau}(\gamma)=\bar{\tau}(\alpha \beta)^{-1}$ is

$$
A(B+x)+A^{-1}\left(B^{-1}-x\right)=\varepsilon\left(C+C^{-1}\right),
$$

hence

$$
x=\frac{\varepsilon\left(C+C^{-1}\right)-A B-(A B)^{-1}}{A-A^{-1}}
$$

is uniquely determined by $A, B, C$, and $\varepsilon$. Let $v:=(B+x)\left(B^{-1}-x\right)$. Since $\bar{\tau}(\beta) \in \operatorname{SL}(2, \mathbb{R})$, we have $y z=v-1$. If $v \neq 1$, then any pair $(y, z)$ of reals with product $\nu-1$ can be obtained by conjugating $\bar{\tau}(\alpha)$ and $\bar{\tau}(\beta)$ by a diagonal matrix in $\operatorname{PGL}(2, \mathbb{R})$ (which does not change $x$ ). Thus $\tau$ is unique up to conjugation once we fix $\varepsilon \in\{-1,1\}$. If $v=1$, then $\bar{\tau}(\beta)$ is either upper or lower triangular, or both, hence there are three conjugacy classes for $\tau$, with $\tau(\Gamma)$ consisting respectively of upper triangular, lower triangular, and diagonal matrices. The condition $v=1$ amounts to $\left(B^{-1}-B-x\right) x=0$, or equivalently to

$$
\left(\frac{B C}{A}-\varepsilon\right)\left(\frac{A C}{B}-\varepsilon\right) \cdot\left(\frac{A B}{C}-\varepsilon\right)(A B C-\varepsilon)=0 ;
$$

in other words, $\varepsilon=1$ and one of $a, b, c$ is the sum of the other two.

Let $j \in \operatorname{Hom}(\Gamma, \operatorname{PSL}(2, \mathbb{R}))$ be geometric (Definition 2.9). For any folding $\rho$ of $j$ along a triskelion lamination $\Upsilon$ of $\Sigma$, the functions $\lambda_{j}$ and $\lambda_{\rho}$ agree on $\{\alpha, \beta, \gamma\}$ (Remark 2.8), and $\rho$ is not conjugate to $j$ under $\operatorname{PGL}(2, \mathbb{R})$ because the folding map $f$ is not an isometry (see Section $2 \mathrm{~A}$ ). Therefore, eu $(\rho)=0$ by the above discussion.

If none of $a, b, c$ is the sum of the other two, then $\rho$ belongs to the unique conjugacy class of representations $\tau$ satisfying (3-2) and eu $(\tau)=0$.

If one of $a, b, c$ is the sum of the other two, then $\rho$ belongs to one of the two conjugacy classes of representations $\tau$ whose image is not virtually abelian and that satisfy (3-2) and $\varepsilon=1$ (Observation 2.7). The representation $\rho^{\prime}$ obtained from $j$ by folding along the image of $\Upsilon$ under the natural involution of the pair of pants 
belongs to the other conjugacy class of such representations. The abelianization of $\rho$ or $\rho^{\prime}$ is not conjugate to $j$, hence satisfies (3-2) and $\varepsilon=1$ as well.

Corollary 3.7. Let $\Gamma=\langle\alpha, \beta, \gamma \mid \alpha \beta \gamma=1\rangle$ be the fundamental group of a pair of pants $\Sigma$, with $\alpha, \beta, \gamma$ corresponding to the three boundary curves. Consider two representations $j, \rho \in \operatorname{Hom}(\Gamma, \operatorname{PSL}(2, \mathbb{R}))$ with $j$ geometric (Definition 2.9), with $\rho$ nongeometric, and with

$$
\left(\lambda_{j}(\alpha), \lambda_{j}(\beta), \lambda_{j}(\gamma)\right)=\left(\lambda_{\rho}(\alpha), \lambda_{\rho}(\beta), \lambda_{\rho}(\gamma)\right)
$$

Then there exists a 1-Lipschitz, $(j, \rho)$-equivariant map $f: \mathbb{M}^{2} \rightarrow \mathbb{M}^{2}$ such that $\operatorname{Lip}_{p}(f)<1$ for any $p \in \mathbb{M}^{2}$ projecting to a point of $j(\Gamma) \backslash \mathbb{\mathbb { M } ^ { 2 }}$ off the boundary of the convex core.

Note that in this setting any $(j, \rho)$-equivariant map $f: \mathbb{H}^{2} \rightarrow \mathbb{Q}^{2}$ satisfies $\operatorname{Lip}(f) \geq 1$ by Remark 2.6, and if $\operatorname{Lip}(f)=1$ then $f$ is an isometry in restriction to the translation axes of $j(\alpha), j(\beta), j(\gamma)$ in $\mathbb{W}^{2}$. The convex core of $j(\Gamma) \backslash \mathbb{Q}^{2}$ naturally identifies with $\Sigma$.

Proof. We first assume that the group $\rho(\Gamma)$ is nonabelian. By Lemma 3.6, the representation $\rho$ is obtained from $j$ by folding along any of at least four of the eight triskelion laminations of $\Sigma$. Let $\ell$ be an injectively immersed geodesic that spirals between two boundary components.

If the two boundary components are different, then $\ell$ is contained in only two triskelion laminations, and intersects the others transversely. If the two boundary components are the same, then $\ell$ intersects transversely all triskelion laminations of $\Sigma$. In both cases we see that a lift of $\ell$ to $\mathbb{H}^{2}$ cannot be isometrically preserved by all 1-Lipschitz, $(j, \rho)$-equivariant maps $f: \mathbb{H}^{2} \rightarrow \mathbb{U}^{2}$ (such maps exist since $\rho$ is a folding of $j$ ). This holds for any $\ell$, which shows that the lamination $\widetilde{\Lambda} \subset \mathbb{H}^{2}$ of Lemma 2.2 is contained in (in fact, is equal to) the preimage of the boundary of the convex core of $j(\Gamma) \backslash \mathbb{M}^{2}$, which identifies with the boundary of $\Sigma$. By Lemma 2.2, this means that there exists a 1-Lipschitz, $(j, \rho)$-equivariant map $f: \mathbb{W}^{2} \rightarrow \mathbb{U}^{2}$ such that $\operatorname{Lip}_{p}(f)<1$ for any $p \in \mathbb{M}^{2}$ projecting to a point of $j(\Gamma) \backslash \mathbb{Q}^{2}$ off the boundary of the convex core.

We now assume that $\rho(\Gamma)$ is abelian. By Lemma 3.6, the representation $\rho$ is the abelianization of some representation $\rho^{\prime}$ that is a folding of $j$. The group $\rho^{\prime}(\Gamma)$ fixes a point $\xi \in \partial_{\infty} \mathbb{H}^{2}$, and $\rho(\Gamma)$ preserves a geodesic line $\ell$ of $\mathbb{M}^{2}$ with endpoint $\xi$. By postcomposing any 1-Lipschitz, $\left(j, \rho^{\prime}\right)$-equivariant map with the projection onto $\ell$ along the horospheres centered at $\xi$, we obtain a 1-Lipschitz, $(j, \rho)$-equivariant map. Moreover, since 1 is the optimal Lipschitz constant (Remark 2.6), this shows that the stretch locus (Definition 2.3) of $(j, \rho)$ is contained in that of $\left(j, \rho^{\prime}\right)$, and we conclude as above. 
Remark 3.8. The nonabelian, nongeometric representations in Lemma 3.6 can also be obtained by folding along a nonmaximal geodesic lamination consisting of a unique leaf spiraling from a boundary component to itself. Folding along a maximal lamination which is not a triskelion gives a representation with values in $\operatorname{PGL}(2, \mathbb{R})$ and not $\operatorname{PSL}(2, \mathbb{R})$.

3C. Proof of Proposition 3.1. By Lemma 3.2, there is a pants decomposition $\Pi$ of $\Sigma_{g}$ such that $\rho$ maps any cuff to a hyperbolic element, and such that if $\rho\left(\Gamma_{g}\right)$ is not virtually abelian then the restriction of $\rho$ to the fundamental group of any pair of pants is nonabelian. Let $j \in \operatorname{Hom}\left(\Gamma_{g}, \operatorname{PSL}(2, \mathbb{R})\right)$ be a Fuchsian representation such that $\lambda_{j}(\gamma)=\lambda_{\rho}(\gamma)$ for all $\gamma \in \Gamma_{g}$ corresponding to cuffs of pants of $\Pi$. The twist parameters along the cuffs will be adjusted later; for the moment we choose them arbitrarily.

Let $\mathscr{C}$ be the $j\left(\Gamma_{g}\right)$-invariant (disjoint) union of all geodesics of $\mathbb{Q}^{2}$ projecting to the cuffs in $j\left(\Gamma_{g}\right) \backslash \mathbb{\boxplus}^{2} \simeq \Sigma_{g}$. For each pair of pants $P$ in $\Pi$, choose a subgroup $\Gamma^{P}$ of $\Gamma_{g}$ which is conjugate to $\pi_{1}(P)$. Then $\left.j\right|_{\Gamma^{P}}$ is the holonomy of a hyperbolic metric on $P$ with cuff lengths given by $\lambda_{\rho}$. Choose a lift $\widetilde{P} \subset \mathbb{M}^{2}$ of the convex core of $j\left(\Gamma^{P}\right) \backslash \mathbb{M}^{2}$. This lift is the closure of a connected component of $\mathbb{M}^{2} \backslash \mathscr{C}$. If the restrictions of $j$ and $\rho$ to $\Gamma^{P}$ are conjugate by some isometry $f^{P}$ of $\mathbb{H}^{2}$, then we give $P$ the label -1 or 1 , depending on whether $f^{P}$ preserves the orientation or not. If the restrictions of $j$ and $\rho$ to $\Gamma^{P}$ are not conjugate, then we give $P$ the label 0. In this case:

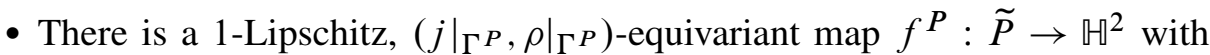
$\operatorname{Lip}_{p}\left(f^{P}\right)<1$ for all $p \notin \partial \widetilde{P}$, by Corollary 3.7.

- If $\rho\left(\Gamma_{g}\right)$ is not virtually abelian then $\left.\rho\right|_{\Gamma^{P}}$ is a folding of $\left.j\right|_{\Gamma^{P}}$ along some triskelion lamination of $P$, by Lemma 3.6; we denote by $F^{P}: \widetilde{P} \rightarrow \mathbb{H}^{2}$ the folding map.

Note that in restriction to any connected component of $\partial \widetilde{P}$ (a line), the maps $f^{P}$ and $F^{P}$ are both isometries, but they may disagree by a constant shift.

The collection of all maps $f^{P}$, extended $(j, \rho)$-equivariantly, piece together to yield a map $f^{*}: \mathbb{M}^{2} \backslash \mathscr{C} \rightarrow \mathbb{M}^{2}$. The obstruction to extending $f^{*}$ by continuity on each geodesic $\ell \subset \mathscr{C}$ is that the maps on either side of $\ell$ may disagree by a constant shift along $\ell$. This discrepancy $\delta(\ell) \in \mathbb{R}$ is the same on the whole $j\left(\Gamma_{g}\right)$ orbit of $\ell$. To correct it, we postcompose $j$ with an earthquake supported on the cuff associated with $\ell$, of length $-\delta(\ell)$. We repeat for each $j\left(\Gamma_{g}\right)$-orbit in $\mathscr{b}$, and eventually obtain a new Fuchsian representation $j_{0}$. By construction, there is a 1Lipschitz, $\left(j_{0}, \rho\right)$-equivariant map $f: \mathbb{M}^{2} \rightarrow \mathbb{M}^{2}$, obtained simply by gluing together isometric translates of the $f^{P}$. This extension $f$ satisfies Proposition 3.1(1).

If $\rho\left(\Gamma_{g}\right)$ is not virtually abelian, then similarly the maps $f^{P}$ for $P$ labeled \pm 1 and $F^{P}$ for $P$ labeled 0 piece together to yield a map $F^{*}: \mathbb{M}^{2} \backslash \mathscr{C} \rightarrow \mathbb{M}^{2}$. As above, 
we can modify $j$ by earthquakes into a new Fuchsian representation $j_{0}^{\prime}$, and $F^{*}$ by piecewise isometries into a $\left(j_{0}^{\prime}, \rho\right)$-equivariant, continuous map $F: \mathbb{T}^{2} \rightarrow \mathbb{M}^{2}$ which is a folding map. This proves Proposition 3.1(2).

Proposition 3.1(3) is satisfied by construction.

3D. Uniform Lipschitz bounds. In order to prove the second claim of Theorem 1.1 in Section 4D, we shall use the following result, which gives Lipschitz bounds which are analogous to Proposition 3.1(1) but uniform.

Proposition 3.9. For any decomposition $\Pi$ of $\Sigma_{g}$ into pairs of pants labeled $-1,0,1$ and any continuous family $\left(j_{t}\right)_{t \geq 0} \subset \operatorname{Hom}\left(\Gamma_{g}, \operatorname{PSL}(2, \mathbb{R})\right)$ of Fuchsian representations, there exist a family $\left(\rho_{t}\right)_{t \geq 0} \subset \operatorname{Hom}\left(\Gamma_{g}, \operatorname{PSL}(2, \mathbb{R})\right)$ of non-Fuchsian representations and, for any $t$ in a small interval $\left[0, t_{0}\right]$, a 1-Lipschitz, $\left(j_{t}, \rho_{t}\right)$ equivariant map $\varphi_{t}: \mathbb{U}^{2} \rightarrow \mathbb{U}^{2}$, with the following properties:

- $\varphi_{t}$ is an orientation-preserving (resp. orientation-reversing) isometry in restriction to any connected subset of $\mathbb{\boxplus}^{2}$ projecting to a union of pants labeled -1 (resp. 1) in $j_{t}\left(\Gamma_{g}\right) \backslash \mathbb{\boxplus}^{2} \simeq \Sigma_{g}$;

- For any $\eta>0$, there exists $C<1$ such that $\operatorname{Lip}_{p}\left(\varphi_{t}\right) \leq C$ for all $t \in\left[0, t_{0}\right]$ and all $p \in \mathbb{U}^{2}$ whose image in $j_{t}\left(\Gamma_{g}\right) \backslash \mathbb{W}^{2} \simeq \Sigma_{g}$ lies inside a pair of pants $P$ labeled 0 , at distance at least $\eta$ from the boundary of $P$.

Proposition 3.9 is based on the following uniform version of Corollary 3.7:

Lemma 3.10. Let $\Gamma=\langle\alpha, \beta, \gamma \mid \alpha \beta \gamma=1\rangle$ be the fundamental group of a pair of pants $\Sigma$, with $\alpha, \beta, \gamma$ corresponding to the three boundary curves. Consider two continuous families $\left(j_{t}\right)_{t \geq 0},\left(\rho_{t}\right)_{t \geq 0} \subset \operatorname{Hom}(\Gamma, \operatorname{PSL}(2, \mathbb{R}))$ of representations with $j_{t}$ geometric (Definition 2.9), with $\rho_{t}$ nongeometric, and with

$$
\left(\lambda_{j_{t}}(\alpha), \lambda_{j_{t}}(\beta), \lambda_{j_{t}}(\gamma)\right)=\left(\lambda_{\rho_{t}}(\alpha), \lambda_{\rho_{t}}(\beta), \lambda_{\rho_{t}}(\gamma)\right)
$$

for all $t \geq 0$. Then there exists a family of 1-Lipschitz, $\left(j_{t}, \rho_{t}\right)$-equivariant maps $\varphi_{t}: \mathbb{U}^{2} \rightarrow \mathbb{U}^{2}$, defined for all $t$ in a small interval $\left[0, t_{0}\right]$, with the following property: for any $\eta>0$ there exists $C<1$ such that $\operatorname{Lip}_{p}\left(\varphi_{t}\right) \leq C$ for all $t \in\left[0, t_{0}\right]$ and all $p \in \mathbb{M}^{2}$ whose image in $j_{t}(\Gamma) \backslash \mathbb{Q}^{2}$ lies at distance at least $\eta$ from the boundary of the convex core.

Proof of Lemma 3.10. By Corollary 3.7, there exists a 1-Lipschitz, $\left(j_{0}, \rho_{0}\right)$-equivariant map $f_{0}: \mathbb{W}^{2} \rightarrow \mathbb{T}^{2}$ such that $\operatorname{Lip}_{p}\left(f_{0}\right)<1$ for any $p \in \mathbb{T}^{2}$ whose image in $j_{0}(\Gamma) \backslash \mathbb{W}^{2}$ does not belong to the boundary of the convex core. If $\left(j_{t}, \rho_{t}\right)=\left(j_{0}, \rho_{0}\right)$ for all $t$, then we may take $\varphi_{t}=f_{0}$. In the general case, we shall build $\varphi_{t}$ as a small

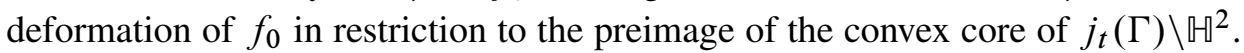

Choose $\Delta>0$ so that for all small $t \geq 0$, the $2 \Delta$-neighborhoods of the boundary components of the convex core of the hyperbolic surface $j_{t}(\Gamma) \backslash \mathbb{M}{ }^{2}$ are disjoint. 


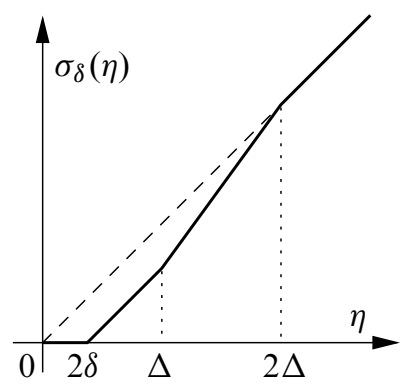

Figure 2. The function $\sigma_{\delta}$ in the proof of Lemma 3.10.

Choose a small $\delta \in(0, \Delta / 2)$ and let $\sigma_{\delta}: \mathbb{R}^{+} \rightarrow \mathbb{R}^{+}$be the function that satisfies

$$
\sigma_{\delta}(\eta)= \begin{cases}0 & \text { for } 0 \leq \eta \leq 2 \delta, \\ \Delta-2 \delta & \text { for } \eta=\Delta, \\ \eta & \text { for } \eta \geq 2 \Delta\end{cases}
$$

and is affine on $[2 \delta, \Delta]$ and $[\Delta, 2 \Delta]$ (Figure 2). Note that $\sigma_{\delta}$ is $(1+o(1))$-Lipschitz as $\delta \rightarrow 0$, and 1-Lipschitz away from $[\Delta, 2 \Delta]$. For any $t \geq 0$, let $N_{t} \subset \mathbb{H}^{2}$ be the preimage of the convex core of $j_{t}(\Gamma) \backslash \mathbb{M}^{2}$, and let $\pi_{t}: \mathbb{W}^{2} \rightarrow N_{t}$ be the closest-point projection, which is 1-Lipschitz. We set

$$
\varphi_{0}:=f_{0} \circ J_{\delta} \circ \pi_{0},
$$

where $J_{\delta}$ is the homotopy of $\mathbb{M}^{2}$ taking any point at distance $\eta \leq 2 \Delta$ from a boundary component $\ell_{0}$ of $N_{0}$ to the point at distance $\sigma_{\delta}(\eta)$ from $\ell_{0}$ on the same perpendicular ray to $\ell_{0}$, leaving other points unchanged. By construction, in restriction to the $2 \delta$-neighborhood of $\partial N_{0}$, the map $\varphi_{0}$ factors through the closest-point projection onto $\partial N_{0}$. The function $p \mapsto \operatorname{Lip}_{p}\left(f_{0}\right)$ is $j_{0}(\Gamma)$-invariant, upper semicontinuous, and less than 1 on $\mathbb{H}^{2} \backslash \partial N_{0}$, hence bounded away from 1 when $p \in N_{0}$ stays at distance at least $\Delta-2 \delta$ from $\partial N_{0}$. This implies that, if we have chosen $\delta$ small enough (which we shall assume from now on), then $\operatorname{Lip}\left(\varphi_{0}\right)=1$ and $\operatorname{Lip}_{p}\left(\varphi_{0}\right)<1$ for all $p$ in the interior of $N_{0}$. For $t>0$, we construct $\varphi_{t}$ as a deformation of $\varphi_{0}$ via a partition of unity, as follows.

Let $u_{t}^{\delta} \subset N_{t}$ be the $\delta$-neighborhood of $\partial N_{t}$ and $N_{t}^{\delta}:=N_{t} \backslash U_{t}^{\delta}$ its complement in $N_{t}$; we define $u_{t}^{2 \delta}$ similarly. Choose a 1-Lipschitz, $\left(j_{t}, \rho_{t}\right)$-equivariant map $\varphi_{t}^{0}: \mathcal{U}_{t}^{2 \delta} \rightarrow \mathbb{M}^{2}$ factoring through the closest-point projection onto $\partial N_{t}$ and taking any boundary component $\ell_{t}$ of $N_{t}$, stabilized by a cyclic subgroup $j_{t}(S)$ of $j_{t}(\Gamma)$, isometrically to the translation axis of $\rho_{t}(S)$ in $\mathbb{H}^{2}$. Up to postcomposing each $\varphi_{t}^{0}$ with an appropriate shift along the axis of $\rho_{t}(S)$, we may assume that $\varphi_{t}^{0}(p) \rightarrow \varphi_{0}(p)$ for any $p \in \mathcal{U}_{0}^{2 \delta}$ as $t \rightarrow 0$ (recall that the restriction of $\varphi_{0}$ to any boundary component of $N_{0}$ is an isometry). 
Let $B^{1}, \ldots, B^{n} \subset N_{0}$ be balls of $\mathbb{W}^{2}$, each projecting injectively to $j_{0}(\Gamma) \backslash \mathbb{M} \mathbb{2}^{2}$, disjoint from a neighborhood of $\partial N_{0}$, and such that

$$
N_{0}^{\delta} \subset j_{0}(\Gamma) \cdot \bigcup_{i=1}^{n} B^{i}
$$

For $1 \leq i \leq n$, let $\varphi_{t}^{i}: j_{t}(\Gamma) \cdot B^{i} \rightarrow \mathbb{H}^{2}$ be the $\left(j_{t}, \rho_{t}\right)$-equivariant map that agrees with $\varphi_{0}$ on $B^{i}$. By construction, for all $1 \leq i \leq n$ (resp. for $i=0$ ) and for all $p \in j_{0}(\Gamma) \cdot B^{i}$ (resp. $p \in \mathcal{U}_{0}^{2 \delta}$ ) we have $\varphi_{t}^{i}(p) \rightarrow \varphi_{0}(p)$ as $t \rightarrow 0$, uniformly for $p$ in any compact set. However, the maps $\varphi_{t}^{i}$, for $0 \leq i \leq n$, may not agree at points where their domains overlap. The goal is to paste them together by the procedure described in Section 2A, using a $j_{t}(\Gamma)$-invariant partition of unity $\left(\psi_{t}^{i}\right)_{0 \leq i \leq n}$ that we now construct.

Let $\psi_{t}^{0}: \mathbb{T}^{2} \rightarrow[0,1]$ be the function supported on $\varkappa_{t}^{2 \delta}$ that takes any point at distance $\eta$ from $\partial N_{t}$ to $\tau(\eta) \in[0,1]$, where $\tau([0, \delta])=1, \tau([2 \delta,+\infty))=0$, and $\tau$ is affine on $[\delta, 2 \delta]$. Let $\psi^{1}, \ldots, \psi^{n}: \mathbb{U}^{2} \rightarrow[0,1]$ be $j_{0}(\Gamma)$-invariant Lipschitz functions inducing a partition of unity on a neighborhood of $N_{0}^{\delta}$, with $\psi^{i}$ supported in $j_{0}(\Gamma) \cdot B^{i}$. Since $N_{t}$ has a compact fundamental domain for $j_{t}(\Gamma)$ that varies continuously with $t$ (for instance a right-angled octagon), for small enough $t$ we have

$$
N_{t}^{\delta} \subset j_{t}(\Gamma) \cdot \bigcup_{i=1}^{n} B^{i}
$$

For $1 \leq i \leq n$ and $t \geq 0$, let $\widehat{\psi}_{t}^{i}: \mathbb{T}^{2} \rightarrow[0,1]$ be the $j_{t}(\Gamma)$-invariant function supported on $j_{t}(\Gamma) \cdot B^{i}$ that agrees with $\psi^{i}$ on $B^{i}$. Then $\sum_{i=1}^{n} \widehat{\psi}_{t}^{i}=1+o(1)$ as $t \rightarrow 0$, with an error term uniform on $N_{t}^{\delta}$. Therefore the functions

$$
\psi_{t}^{0} \quad \text { and } \quad \psi_{t}^{i}:=\left(1-\psi_{t}^{0}\right) \frac{\widehat{\psi}_{t}^{i}}{\sum_{k=1}^{n} \widehat{\psi}_{t}^{k}}: \mathbb{T}^{2} \longrightarrow[0,1]
$$

for $1 \leq i \leq n$ form a $j_{t}(\Gamma)$-invariant partition of unity of $N_{t}$, subordinated to the covering $\mathcal{U}_{t}^{2 \delta} \cup j_{t}(\Gamma) \cdot B^{1} \cup \cdots \cup j_{t}(\Gamma) \cdot B^{n} \supset N_{t}$, and are all $L$-Lipschitz for some $L>0$ independent of $i$ and $t$.

For $t \geq 0$, let $\varphi_{t}:=\sum_{i=0}^{n} \psi_{t}^{i} \varphi_{t}^{i}: N_{t} \rightarrow \mathbb{H}^{2}$ be the averaged map defined in Section 2A. This map is $\left(j_{t}, \rho_{t}\right)$-equivariant by construction. We extend it to a map $\varphi_{t}: \mathbb{H}^{2} \rightarrow \mathbb{M}^{2}$ by precomposing with the closest-point projection $\pi_{t}: \mathbb{H}^{2} \rightarrow N_{t}$. We claim that the maps $\varphi_{t}$ satisfy the conclusion of Lemma 3.10. Indeed, by Lemma 2.4, for any $t \geq 0$ and $p$ in the interior of $N_{t}$,

$$
\operatorname{Lip}_{p}\left(\varphi_{t}\right) \leq \sum_{i \in I_{t}(p)}\left(\operatorname{Lip}_{p}\left(\psi_{t}^{i}\right) R_{t}(p)+\psi_{t}^{i}(p) \operatorname{Lip}_{p}\left(\varphi_{t}^{i}\right)\right)
$$


where $I_{t}(p)$ is the set of indices $0 \leq i \leq n$ such that $p$ belongs to the support of $\psi_{t}^{i}$, and $R_{t}(p) \geq 0$ is the diameter of the set $\left\{\varphi_{t}^{i}(p) \mid i \in I_{t}(p)\right\}$. Let $\eta>0$ be the distance from $p$ to $\partial N_{t}$.

If $\eta<\delta$, then $\varphi_{t}$ coincides on a neighborhood of $p$ with $\varphi_{t}^{0}$, hence with the closest-point projection onto $\partial N_{t}$ postcomposed with an isometry of $\mathbb{H}^{2}$, and the right-hand side of (3-3) reduces to

$$
\operatorname{Lip}_{p}\left(\varphi_{t}^{0}\right)=\frac{1}{\cosh \eta}<1
$$

(see [Guéritaud and Kassel 2013, (A.9)], for instance).

If $\eta \geq \delta$, then the bound on $\operatorname{Lip}_{p}\left(\varphi_{t}^{0}\right)$ still holds, and $\operatorname{Lip}_{p}\left(\varphi_{t}^{i}\right)$ for $1 \leq i \leq n$ can also be uniformly bounded away from 1 . Indeed, $\sup _{q \in B^{i}} \operatorname{Lip}_{q}\left(\varphi_{t}^{i}\right)<1$ since $B^{i}$ is disjoint from a neighborhood of $\partial N_{0}$ and the local Lipschitz constant is upper semicontinuous, and we argue by equivariance. Moreover, all the other contributions to (3-3) are small: $R_{t}(p) \rightarrow 0$ as $t \rightarrow 0$, uniformly in $p$, and $\operatorname{Lip}_{p}\left(\psi_{t}^{i}\right)$ is bounded independently of $p, i, t$ (by $L$ ). Therefore, for small $t$ there exists $C<1$, independent of $p$ and $t$, such that $\operatorname{Lip}_{p}\left(\varphi_{t}\right) \leq C$.

This treats the case when $p \in N_{t}$. To conclude, we note that on a neighborhood of any $p \in \mathbb{T}^{2} \backslash N_{t}$ the map $\varphi_{t}$ coincides with the closest-point projection onto $\partial N_{t}$ postcomposed with an isometry of $\mathbb{U}^{2}$, hence $\operatorname{Lip}_{p}\left(\varphi_{t}\right)=1 / \cosh \eta<1$, where $\eta=d\left(p, \partial N_{t}\right)$.

Proof of Proposition 3.9. Let $\Upsilon$ be a lamination of $\Sigma_{g}$ consisting of all the cuffs of $\Pi$ together with a triskelion lamination inside each pair of pants labeled 0 . Let $c: \Sigma_{g} \backslash \Upsilon \rightarrow\{-1,1\}$ be a coloring taking the value -1 (resp. 1) on each pair of pants labeled -1 (resp. 1), and both values on each pair of pants labeled 0 . For any $t \geq 0$, let $\rho_{t}^{\prime}$ be the folding of $j_{t}$ along $\Upsilon$ with coloring $c$.

We now argue similarly to the proof of Proposition 3.1 in Section 3C. For each pair of pants $P$ in $\Pi$, choose a subgroup $\Gamma^{P}$ of $\Gamma_{g}$ which is conjugate to $\pi_{1}(P)$, and for any $t \geq 0$ a lift $\widetilde{P}_{t} \subset \mathbb{U}^{2}$ of the convex core of $j_{t}\left(\Gamma^{P}\right) \backslash \mathbb{H}^{2}$.

If $P$ is labeled -1 (resp. 1), then for any $t \geq 0$ the restrictions of $j_{t}$ and $\rho_{t}^{\prime}$ to $\Gamma^{P}$ are conjugate by some orientation-preserving (resp. orientation-reversing) isometry $\varphi_{t}^{P}$ of $\mathbb{T}^{2}$.

If $P$ is labeled 0 , then, by Lemma 3.10, there is a family of 1-Lipschitz, $\left(\left.j_{t}\right|_{\Gamma^{P}},\left.\rho_{t}^{\prime}\right|_{\Gamma^{P}}\right)$-equivariant maps $\varphi_{t}^{P}: \mathbb{M}^{2} \rightarrow \mathbb{H}^{2}$, defined for all $t$ in a small interval $\left[0, t_{0}\right]$, with the following property: for any $\eta>0$, there exists $C<1$ such that $\operatorname{Lip}_{p}\left(\varphi_{t}^{P}\right) \leq C$ for all $t \in\left[0, t_{0}\right]$ and all $p \in \widetilde{P}_{t}$ at distance at least $\eta$ from $\partial \widetilde{P}_{t}$.

The collection of all maps $\varphi_{t}^{P}$, extended $\left(j_{t}, \rho_{t}^{\prime}\right)$-equivariantly, piece together to yield a map $\varphi_{t}^{*}: \mathbb{W}^{2} \backslash \mathscr{C}_{t} \rightarrow \mathbb{U}^{2}$, where $\mathscr{C}_{t}$ is the union of all geodesics of $\mathbb{W}^{2}$ projecting to cuffs of $\Pi$ in $j_{t}\left(\Gamma_{g}\right) \backslash \mathbb{W}^{2} \simeq \Sigma_{g}$. 
The obstruction to extending $\varphi_{t}^{*}$ by continuity on each geodesic $\ell_{t} \subset \mathscr{b}_{t}$ is that the maps on either side of $\ell_{t}$ may disagree by a constant shift along $\ell_{t}$ if $\ell_{t}$ separates two pairs of pants labeled $( \pm 1,0)$ or $(0,0)$. This discrepancy $\delta\left(\ell_{t}\right) \in \mathbb{R}$ is the same on the whole $j_{t}\left(\Gamma_{g}\right)$-orbit of $\ell_{t}$. To correct it, we precompose the folding $\rho_{t}^{\prime}$ of $j_{t}$ with an earthquake, supported on the cuff associated with $\ell_{t}$ (in the $j_{t}$-metric), of length $-\delta\left(\ell_{t}\right)$. We repeat for each $j_{t}\left(\Gamma_{g}\right)$-orbit in $\mathscr{C}_{t}$, and eventually obtain a new folded representation $\rho_{t}$. By construction, there is a family of 1-Lipschitz, $\left(j_{t}, \rho_{t}\right)$-equivariant maps $\varphi_{t}: \mathbb{U}^{2} \rightarrow \mathbb{W}^{2}$ satisfying Proposition 3.9, obtained simply by gluing together isometric translates of the $\varphi_{t}^{P}$.

\section{Surjectivity of the two projections}

In this section we prove Theorem 1.1. We first construct uniformly lengthening deformations of surfaces with boundary (Section 4A), then glue these together according to combinatorics given by Proposition 3.1 (Sections 4B and 4D). Section 4C is devoted to the proof of a technical lemma.

4A. Uniformly lengthening deformations of compact hyperbolic surfaces with boundary. Our two main tools to prove Theorem 1.1 are Proposition 3.1 and the following lemma:

Lemma 4.1. Let $\Gamma$ be the fundamental group and $j_{0} \in \operatorname{Hom}(\Gamma, \operatorname{PSL}(2, \mathbb{R}))$ the holonomy of a compact, connected, hyperbolic surface $\Sigma$ with nonempty geodesic boundary. Then there exist $t_{0}>0$ and a continuous family of representations $\left(j_{t}\right)_{0 \leq t \leq t_{0}}$ with the following properties:

(a) $\lambda_{j_{0}}(\gamma)=(1-t) \lambda_{j_{t}}(\gamma)$ for any $t \in\left[0, t_{0}\right]$ and any $\gamma \in \Gamma$ corresponding to a boundary component of $\Sigma$;

(b) $\sup _{\gamma \in \Gamma \backslash\{1\}} \lambda_{j_{0}}(\gamma) / \lambda_{j_{t}}(\gamma)<1$ for any $t \in\left(0, t_{0}\right]$;

(c) $j_{t}(\gamma)=j_{0}(\gamma)+O(t)$ for any $\gamma \in \Gamma$ as $t \rightarrow 0$, where both sides are seen as $2 \times 2$ real matrices with determinant 1 modulo $\pm \mathrm{Id}$;

(d) for any compact subset $K$ of $\mathbb{M}^{2}$ projecting to the interior of the convex core of $j_{0}(\Gamma) \backslash \mathbb{M}^{2}$, there exists $L>0$ such that

$$
d\left(p, f_{t}(p)\right) \leq L t
$$

for any $p \in K$, any $t \in\left[0, t_{0}\right]$, and any 1-Lipschitz, $\left(j_{t}, j_{0}\right)$-equivariant map $f_{t}: \mathbb{U}^{2} \rightarrow \mathbb{U}^{2}$.

As in Section 3B, the convex core of $j_{0}(\Gamma) \backslash \mathbb{M}^{2}$ naturally identifies with $\Sigma$. The idea is to construct the representations $j_{t}$ as holonomies of hyperbolic surfaces obtained from $j_{0}(\Gamma) \backslash \mathbb{T}^{2}$ by strip deformations. This type of deformation was first 


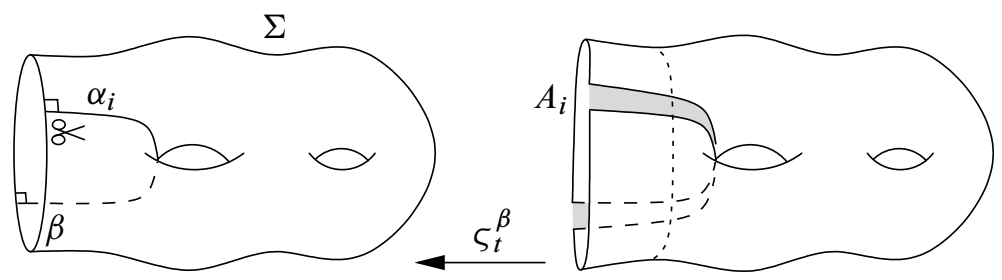

Figure 3. A strip deformation. In the source of the collapsing map $\varsigma_{t}^{\beta}$ we show the new peripheral geodesic, dotted.

introduced by Thurston [1986, proof of Lemma 3.4]. We refer to [Papadopoulos and Théret 2010; Danciger et al. 2014] for more details.

Proof. We first explain how to lengthen one boundary component $\beta$ of $\Sigma$. Choose a finite collection of disjoint, biinfinite geodesic $\operatorname{arcs} \alpha_{1}, \ldots, \alpha_{n} \subset j_{0}(\Gamma) \backslash \mathbb{Q}^{2}$, each crossing $\beta$ orthogonally twice, that subdivide the convex core $\Sigma$ into rightangled hexagons and one-holed right-angled bigons. Along each $\operatorname{arc} \alpha_{i}$, following [Thurston 1986], slice $j_{0}(\Gamma) \backslash \mathbb{U}^{2}$ open and insert a strip $A_{i}$ of $\mathbb{W}^{2}$, bounded by two geodesics, with narrowest cross-section at the midpoint of $\alpha_{i} \cap \Sigma$ (see Figure 3).

This yields a new complete hyperbolic surface, with a compact convex core, equipped with a natural 1-Lipschitz map $\varsigma_{t}^{\beta}$ to $j_{0}(\Gamma) \backslash \mathbb{W}^{2}$ obtained by collapsing the strips $A_{i}$ back to lines. Note that the image under $\varsigma_{t}^{\beta}$ of the new convex core is strictly contained in $\Sigma$ (see Figure 3 ). The geodesic corresponding to $\beta$ is longer in the new surface than in $\Sigma$. By adjusting the widths of the strips $A_{i}$, we may assume that the ratio of lengths is $1 /(1-t)$. The appropriate widths for this ratio are in $O(t)$ as $t \rightarrow 0$. All lengths of geodesics corresponding to boundary components other than $\beta$ are unchanged.

Repeat the construction, iteratively, for all boundary components $\beta_{1}, \ldots, \beta_{r}$ of $\Sigma$, in some arbitrary order. We thus obtain a new complete hyperbolic surface $j_{t}(\Gamma) \backslash \mathbb{Q}^{2}$, with a compact convex core $\Sigma_{t}$, such that $j_{t}$ satisfies (a).

We claim that $j_{t}$ also satisfies (b). Indeed, consider the 1-Lipschitz map $\varsigma_{t}:=\varsigma_{t}^{\beta_{r}} \circ \cdots \circ \varsigma_{t}^{\beta_{1}}$ from $\Sigma_{t}$ to $\Sigma$. If 1 were its optimal Lipschitz constant, then by Lemma 2.2 there would exist a geodesic lamination of $\Sigma_{t}$ whose leaves are isometrically preserved by $\varsigma_{t}$. But this is not the case here since for every $i$, the map $\varsigma_{t}^{\beta_{i}}$ does not isometrically preserve any geodesic lamination except the boundary components other than $\beta_{i}$. Therefore $\varsigma_{t}$ has Lipschitz constant strictly less than 1, which implies (b) by Remark 2.6.

Up to replacing each $j_{t}$ with a conjugate under $\operatorname{PSL}(2, \mathbb{R})$, we may assume that (c) holds. Indeed, it is well known that there exist elements $\gamma_{1}, \ldots, \gamma_{n} \in \Gamma$ whose length functions form a smooth coordinate system for $\operatorname{Hom}(\Gamma, \operatorname{PSL}(2, \mathbb{R})) / \operatorname{PSL}(2, \mathbb{R})$ near $\left[j_{0}\right]$ (see [Goldman and Xia 2011, Theorem 2.1] for instance). For any $i$, the preimage under $\varsigma_{t}$ of the closed geodesic of $\Sigma$ associated with $\gamma_{i}$ is obtained by 

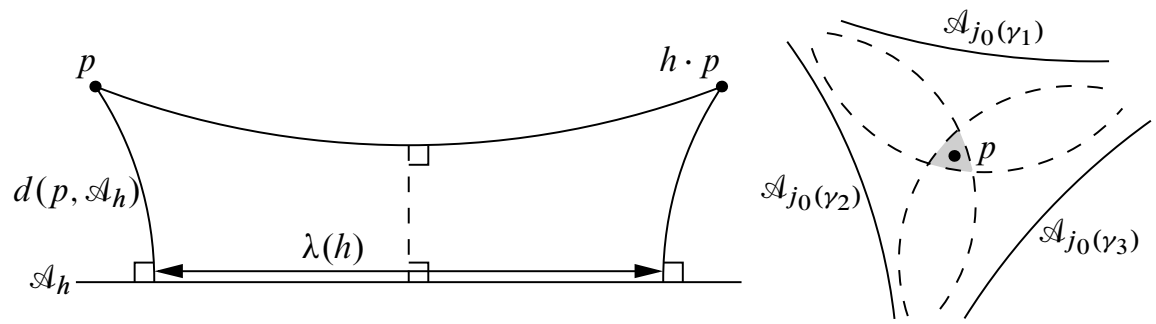

Figure 4. Illustration of the proof of Lemma 4.1. Left: a hyperbolic quadrilateral with two right angles. Right: the point $f_{t}(p)$ belongs to the shaded region.

expanding finitely many strips of width $O(t)$, hence $\lambda_{j_{t}}\left(\gamma_{i}\right) \leq \lambda_{j_{0}}\left(\gamma_{i}\right)+O(t)$ as $t \rightarrow 0$. On the other hand, $\lambda_{j_{t}}\left(\gamma_{i}\right) \geq \lambda_{j_{0}}\left(\gamma_{i}\right)$ due to the existence of the 1-Lipschitz map $\varsigma_{t}$. Therefore, $d^{\prime}\left(j_{0}, j_{t}\right)=O(t)$ for any smooth metric $d^{\prime}$ on a neighborhood of $\left[j_{0}\right]$ in $\operatorname{Hom}(\Gamma, \operatorname{PSL}(2, \mathbb{R})) / \operatorname{PSL}(2, \mathbb{R})$.

To check (d), we use a perturbative version of the argument that a $j_{0}(\Gamma)$-invariant, 1-Lipschitz map must be the identity on the preimage $N_{0} \subset \mathbb{M}^{2}$ of the convex core $\Sigma$ of $j_{0}(\Gamma) \backslash \mathbb{M}^{2}$. For any hyperbolic element $h \in \operatorname{PSL}(2, \mathbb{R})$, with translation axis $\mathscr{A}_{h} \subset \mathbb{H}^{2}$, and for any $p \in \mathbb{H}^{2}$, a classical formula gives

$$
\sinh \left(\frac{1}{2} d(p, h \cdot p)\right)=\sinh \left(\frac{1}{2} \lambda(h)\right) \cdot \cosh d\left(p, \mathscr{A}_{h}\right)
$$

(see Figure 4, left). Consider $p \in \mathbb{H}^{2}$ in the interior of $N_{0}$. We can find three translation axes $\mathscr{A}_{j_{0}\left(\gamma_{1}\right)}, \mathscr{A}_{j_{0}\left(\gamma_{2}\right)}, \mathscr{A}_{j_{0}\left(\gamma_{3}\right)} \subset \partial N_{0}$ of elements of $j_{0}(\Gamma)$ such that, if $q_{i}$ denotes the projection of $p$ to $\mathscr{A}_{j_{0}\left(\gamma_{i}\right)}$, then $p$ belongs to the interior of the triangle $q_{1} q_{2} q_{3}$. For any $t \geq 0$ and any 1-Lipschitz, $\left(j_{t}, j_{0}\right)$-equivariant map $f_{t}: \mathbb{H}^{2} \rightarrow \mathbb{H}^{2}$,

$$
d\left(f_{t}(p), j_{0}\left(\gamma_{i}\right) \cdot f_{t}(p)\right) \leq d\left(p, j_{t}\left(\gamma_{i}\right) \cdot p\right),
$$

which by (4-1) may be written as

$$
\sinh \left(\frac{1}{2} \lambda_{j_{0}}\left(\gamma_{i}\right)\right) \cdot \cosh d\left(f_{t}(p), \mathscr{A}_{j_{0}\left(\gamma_{i}\right)}\right) \leq \sinh \left(\frac{1}{2} \lambda_{j_{t}}\left(\gamma_{i}\right)\right) \cdot \cosh d\left(p, \mathscr{A}_{j_{t}\left(\gamma_{i}\right)}\right) .
$$

Since $\lambda_{j_{0}}\left(\gamma_{i}\right)=\lambda_{j_{t}}\left(\gamma_{i}\right)+O(t)$ and $d\left(p, \mathscr{A}_{j_{t}\left(\gamma_{i}\right)}\right)=d\left(p, \mathscr{A}_{j_{0}\left(\gamma_{i}\right)}\right)+O(t)$ by (c), this implies

$$
\cosh d\left(f_{t}(p), \mathscr{A}_{j_{0}\left(\gamma_{i}\right)}\right) \leq \cosh d\left(p, \mathscr{A}_{j_{0}\left(\gamma_{i}\right)}\right)+O(t),
$$

where the error term does not depend on the choice of $f_{t}$. Since $d\left(p, \mathscr{A}_{j_{0}\left(\gamma_{i}\right)}\right)>0$, we may invert the hyperbolic cosine:

$$
d\left(f_{t}(p), \mathscr{A}_{j_{0}\left(\gamma_{i}\right)}\right) \leq d\left(p, \mathscr{A}_{j_{0}\left(\gamma_{i}\right)}\right)+O(t) .
$$

Applied to $i=1,2,3$, this means that $f_{t}(p)$ belongs to a curvilinear triangle around $p$ bounded by three hypercycles (curves at constant distance from a geodesic 


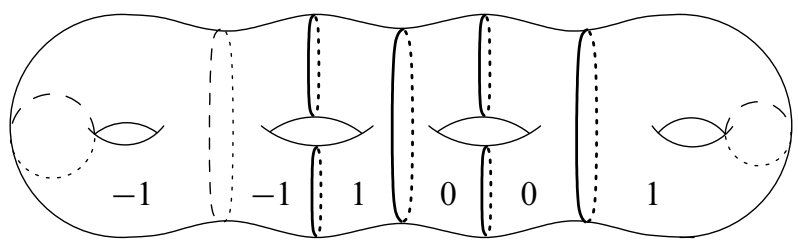

Figure 5. A labeled pants decomposition with $m=5$. The boundary components of the $\Sigma^{i}, 1 \leq i \leq 5$, are in bold.

line) expanding at rate $O(t)$ as $t$ becomes positive, hence $d\left(p, f_{t}(p)\right)=O(t)$ (see Figure 4, right). All estimates $O(t)$ are robust under small perturbations of $p$, hence can be made uniform (and still independent of $f_{t}$ ) for $p$ in a compact set $K$, yielding (d).

4B. Gluing surfaces with boundary. We now prove the first claim of Theorem 1.1. Namely, given $[\rho] \in \operatorname{Rep}_{g}^{\text {nfd }}$, we construct $[j] \in \operatorname{Rep}_{g}^{\text {fd }}$ that strictly dominates $[\rho]$.

If $\lambda_{\rho} \equiv 0$, then any $[j] \in \operatorname{Rep}_{g}^{\mathrm{fd}}$ strictly dominates $[\rho]$. We now suppose $\lambda_{\rho} \not \equiv 0$. Proposition 3.1(1) then gives us an element $\left[j_{0}\right] \in \operatorname{Rep} \mathrm{fd}_{g}^{\mathrm{fd}}$, a labeled pants decomposition $\Pi$ of $\Sigma_{g}$, and, for any $j_{0}, \rho \in \operatorname{Hom}\left(\Gamma_{g}, \operatorname{PSL}(2, \mathbb{R})\right)$ in the respective classes $\left[j_{0}\right],[\rho]$ (which we now fix), a 1-Lipschitz, $\left(j_{0}, \rho\right)$-equivariant map $f: \mathbb{M}^{2} \rightarrow \mathbb{M}^{2}$ that is an orientation-preserving (resp. orientation-reversing) isometry in restriction to any connected subset of $\mathbb{H}^{2}$ projecting to a union of pants labeled -1 (resp. 1) in $j_{0}\left(\Gamma_{g}\right) \backslash \mathbb{U}^{2} \simeq \Sigma_{g}$ and that satisfies $\operatorname{Lip}_{p}(f)<1$ for any $p \in \mathbb{T}^{2}$ projecting to the interior of a pair of pants labeled 0 . Not all pairs of pants are labeled -1 , and not all 1 , since $j_{0}$ and $\rho$ are not conjugate under PGL $(2, \mathbb{R})$. By Remark 2.6, the class $\left[j_{0}\right]$ dominates $[\rho]$ in the sense that $\lambda(\rho(\gamma)) \leq \lambda\left(j_{0}(\gamma)\right)$ for all $\gamma \in \Gamma_{g}$. Our goal is to use Lemma 4.1 to modify $j_{0}$ into a representation $j$ such that $[j]$ strictly dominates $[\rho]$.

For this purpose, we erase all the cuffs that separate two pairs of pants of $\Pi$ with labels $(-1,-1)$ or $(1,1)$, and write

$$
\Sigma_{g}=\Sigma^{1} \cup \cdots \cup \Sigma^{m},
$$

where $\Sigma^{i}$, for any $1 \leq i \leq m$, is a compact surface with boundary that is one of:

- a pair of pants labeled 0 ,

- a full connected component of the subsurface of $\Sigma_{g}$ made of pants labeled -1,

- or a full connected component of the subsurface of $\Sigma_{g}$ made of pants labeled 1;

(see Figure 5). The boundary components of the $\Sigma^{i}$ are the cuffs that separated two pairs of pants of $\Pi$ with labels $(-1,1),( \pm 1,0)$ or $(0,0)$. Choose a small $\delta>0$ such that, in all hyperbolic metrics on $\Sigma_{g}$ which are close enough to that defined by $j_{0}$, any simple geodesic entering the $\delta$-neighborhood of the geodesic representative 
of a cuff of $\Pi$ crosses it. Let $\mathscr{C}_{0} \subset \mathbb{M}^{2}$ be the union of all geodesic lines of $\mathbb{M}^{2}$ projecting to boundary components of the $\Sigma^{i}$ in $j_{0}\left(\Gamma_{g}\right) \backslash \mathbb{\boxplus}^{2} \simeq \Sigma_{g}$, let $N_{0}^{\delta} \subset \mathbb{M}^{2}$ be the complement of the $\delta$-neighborhood of $\mathscr{C}_{0}$, and let $K \subset \mathbb{M}^{2} \backslash \mathscr{C}_{0}$ be a compact set whose interior contains a fundamental domain of $N_{0}^{\delta}$ for the action of $j_{0}\left(\Gamma_{g}\right)$, with $m$ connected components projecting respectively to $\Sigma^{1}, \ldots, \Sigma^{m}$.

We apply Lemma 4.1 to $\Gamma^{i}:=\pi_{1}\left(\Sigma^{i}\right)$ and $j_{0}^{i}:=\left.j_{0}\right|_{\Gamma^{i}}$ and obtain continuous families $\left(j_{t}^{i}\right)_{0 \leq t \leq t_{0}} \subset \operatorname{Hom}\left(\Gamma^{i}, \operatorname{PSL}(2, \mathbb{R})\right)$ of representations for $1 \leq i \leq m$ satisfying properties (a)-(d) of Lemma 4.1 with a uniform constant $L>0$ for the compact set $K \subset \mathbb{H}^{2} \backslash \mathscr{C}_{0}$. For any $t \in\left[0, t_{0}\right]$, using (a), we can glue together the (compact) convex cores of the $j_{t}^{i}\left(\Gamma^{i}\right) \backslash \mathbb{H}^{2}$ following the same combinatorics as the $\Sigma^{i}$. This gives a closed hyperbolic surface of genus $g$, hence a holonomy representation $j_{t} \in \operatorname{Hom}\left(\Gamma_{g}, \operatorname{PSL}(2, \mathbb{R})\right)$. By (c), up to adjusting the twist parameters, we may assume that

$$
j_{t}(\gamma)=j_{0}(\gamma)+O(t)
$$

for any $\gamma \in \Gamma_{g}$ as $t \rightarrow 0$, where both sides are seen as $2 \times 2$ real matrices with determinant 1 modulo \pm Id.

To complete the proof of the first statement of Theorem 1.1, it is sufficient to prove that for small enough $t>0$,

$$
\sup _{\gamma \in\left(\Gamma_{g}\right)_{s}} \frac{\lambda_{\rho}(\gamma)}{\lambda_{j_{t}}(\gamma)}<1
$$

where $\left(\Gamma_{g}\right)_{s}$ is the set of nontrivial elements of $\Gamma_{g}$ corresponding to simple closed curves on $\Sigma_{g}$; then $[j]:=\left[j_{t}\right]$ will strictly dominate $[\rho]$ by Theorem 2.5 . Note that $\lambda\left(j_{t}(\gamma)\right)=\lambda\left(j_{t}^{i}(\gamma)\right)$ for all $\gamma$ in $\Gamma^{i}$, seen as a subgroup of $\Gamma_{g}$. Thus (b) gives the control required in (4-3) for simple closed curves contained in one of the $\Sigma^{i}$. We now explain why the lengths of the other simple closed curves also decrease uniformly, based on (b), (c), and (d).

For any $t \in\left(0, t_{0}\right]$, let $\mathscr{C}_{t} \subset \mathbb{M}^{2}$ be the union of the lifts to $\mathbb{M}^{2}$ of the simple closed geodesics of $j_{t}\left(\Gamma_{g}\right) \backslash \mathbb{M}^{2} \simeq \Sigma_{g}$ corresponding to $\mathscr{C}_{0}$ and let $N_{t}^{\delta}$ be the complement of the $\delta$-neighborhood of $\mathscr{C}_{t}$ in $\mathbb{\boxplus}^{2}$. For $t$ small enough, we can find a fundamental domain $K_{t}$ of $N_{t}^{\delta}$ for the action of $j_{t}\left(\Gamma_{g}\right)$ that is contained in $K$ and has $m$ connected components. By (b) and Theorem 2.5, for any $1 \leq i \leq m$ and $t \in\left(0, t_{0}\right]$ there exists a $\left(\left.j_{t}\right|_{\Gamma^{i}},\left.j_{0}\right|_{\Gamma^{i}}\right)$-equivariant map $f_{t}^{i}: \mathbb{U}^{2} \rightarrow \mathbb{M}^{2}$ with $\operatorname{Lip}\left(f_{t}^{i}\right)<1$. For small $t>0$, we choose a $\left(j_{t}, j_{0}\right)$-equivariant map $f_{t}:\left(N_{t}^{\delta} \cup \mathscr{C}_{t}\right) \rightarrow \mathbb{H}^{2}$ such that:

- $f_{t}=f_{t}^{i}$ on the component of $K_{t}$ projecting to $\Sigma^{i}$ for all $1 \leq i \leq m$;

- $f_{t}$ takes any geodesic line in $\mathscr{C}_{t}$ to the corresponding line in $\mathscr{C}_{0}$, multiplying all distances on it by the uniform factor $(1-t)$. 
We choose the $f_{t}$ so that, in addition, for any compact set $K^{\prime} \subset \mathbb{U}^{2}$ there exists $L_{1} \geq 0$ such that $d\left(x^{\prime}, f_{t}\left(x^{\prime}\right)\right) \leq L_{1} t$ for all small enough $t>0$ and all $x^{\prime} \in \mathscr{C}_{t} \cap K^{\prime}$. Consider the $\left(j_{t}, \rho\right)$-equivariant map

$$
F_{t}:=f \circ f_{t}:\left(N_{t}^{\delta} \cup \mathscr{C}_{t}\right) \longrightarrow \mathbb{M}^{2},
$$

where $f: \mathbb{M}^{2} \rightarrow \mathbb{Q}^{2}$ is the $\left(j_{0}, \rho\right)$-equivariant map from the beginning of the proof. In order to prove (4-3), it is sufficient to establish the following:

Lemma 4.2. For small enough $t>0$, there exists $C<1$ such that for all $p, q \in \partial N_{t}^{\delta}$ lying at distance $\delta$ from a line $\ell_{t} \subset \mathscr{C}_{t}$, on opposite sides of $\ell_{t}$,

$$
d\left(F_{t}(p), F_{t}(q)\right) \leq C d(p, q) .
$$

Indeed, fix a small $t>0$. Any geodesic segment $I=[p, q]$ of $\mathbb{H}^{2}$ projecting to a closed geodesic of $j_{t}\left(\Gamma_{g}\right) \backslash \mathbb{M}^{2} \simeq \Sigma_{g}$ may be decomposed into subsegments $I_{1}, \ldots, I_{n}$ contained in $N_{t}^{\delta}$ alternating with subsegments $I_{1}^{\prime}, \ldots, I_{n}^{\prime}$ crossing connected components of $\mathbb{M}^{2} \backslash N_{t}^{\delta}$ (indeed, any simple closed curve that enters one of these components crosses it, by the choice of $\delta$ ). By construction, the map $F_{t}$ has Lipschitz constant strictly less than 1 on each connected component of $N_{t}^{\delta}$, hence moves the endpoints of each $I_{k}$ closer together by a uniform factor (independent of $I$ ). Lemma 4.2 ensures that the same holds for the $I_{k}^{\prime}$. Thus the ratio $d\left(F_{t}(p), F_{t}(q)\right) / d(p, q)$ is bounded by some factor $C^{\prime}<1$ independent of $I$, and the corresponding element $\gamma \in \Gamma_{g}$ satisfies $\lambda(\rho(\gamma)) \leq C^{\prime} \lambda\left(j_{t}(\gamma)\right)$. This proves (4-3), hence completes the proof of the first statement of Theorem 1.1.

4C. Proof of Lemma 4.2. We first make the following observation:

Observation 4.3. There exists $L^{\prime} \geq 0$ such that, for any small enough $t>0$, any $p \in \partial N_{t}^{\delta}$ at distance $\delta$ from a geodesic $\ell_{t} \subset \mathscr{C}_{t}$, and any $x \in \ell_{t}$,

$$
d\left(f_{t}(p), f_{t}(x)\right) \leq(1-t) d(p, x)+L^{\prime} t .
$$

Proof. Since $f_{t}$ is $\left(j_{t}, j_{0}\right)$-equivariant and $\mathscr{C}_{0}$ has only finitely many connected components modulo $j_{0}\left(\Gamma_{g}\right)$, we may fix a geodesic $\ell_{0} \subset \mathscr{C}_{0}$ and prove the observation only for the geodesics $\ell_{t} \subset \mathscr{C}_{t}$ corresponding to $\ell_{0}$. For any $t>0$, the map $f_{t}$ takes $\ell_{t}$ linearly to $\ell_{0}$, multiplying all distances by the uniform factor $1-t$. Let $h_{t}: \mathbb{\boxplus}^{2} \rightarrow \mathbb{Q}^{2}$ be the orientation-preserving map that coincides with $f_{t}$ on $\ell_{t}$, takes any line orthogonal to $\ell_{t}$ to a line orthogonal to $\ell_{0}$, and multiplies all distances by $1-t$ on such lines. At distance $\eta$ from $\ell_{t}$, the differential of $h_{t}$ has principal values $1-t$ and $(1-t) \cosh ((1-t) \eta) / \cosh \eta \leq 1-t$ (see [Guéritaud and Kassel 2013, (A.9)]), hence $\operatorname{Lip}\left(h_{t}\right) \leq 1-t$ and

$$
d\left(f_{t}(x), h_{t}(p)\right)=d\left(h_{t}(x), h_{t}(p)\right) \leq(1-t) d(x, p)
$$


for all $x \in \ell_{t}$ and $p \in \mathbb{H}^{2}$. By the triangle inequality, it is enough to find $L^{\prime} \geq 0$ such that $d\left(h_{t}(p), f_{t}(p)\right) \leq L^{\prime} t$ for all small enough $t>0$ and all $p \in \partial N_{t}^{\delta}$ at distance $\delta$ from $\ell_{t}$. Since $f_{t}$ and $h_{t}$ are both $\left(j_{t}, j_{0}\right)$-equivariant under the stabilizer $S$ of $\ell_{0}$ in $\Gamma_{g}$, and $j_{t}(S)$ acts cocompactly on the set $\bar{u}_{t}$ of points at distance at most $\delta$ from $\ell_{t}$, we may restrict to $p$ in a compact fundamental domain of $\bar{\varkappa}_{t}$ for $j_{t}(S)$. Let $K^{\prime} \subset \mathbb{H}^{2}$ be a compact set containing such fundamental domains for all $t \in\left[0, t_{0}\right]$. By construction of $f_{t}$, there exists $L_{1} \geq 0$ such that $d\left(x^{\prime}, f_{t}\left(x^{\prime}\right)\right) \leq L_{1} t$ for all small enough $t>0$ and all $x^{\prime} \in \ell_{t} \cap K^{\prime}$. By definition of $h_{t}$, this implies the existence of $L_{2} \geq 0$ such that $d\left(p, h_{t}(p)\right) \leq L_{2} t$ for all small enough $t>0$ and all $p \in K^{\prime}$. On the other hand, condition (d) of Lemma 4.1 (applied to the $\Gamma^{i}$ and $j_{0}^{i}$ as in Section 4B) implies the existence of $L_{3} \geq 0$ such that $d\left(p, f_{t}(p)\right) \leq L_{3} t$ for all $t$ and $p \in \partial N_{t}^{\delta} \cap K^{\prime}$. By the triangle inequality, we may take $L^{\prime}=L_{2}+L_{3}$.

Proof of Lemma 4.2. As in the proof of Observation 4.3, we may fix a geodesic $\ell_{0} \subset \mathscr{C}_{0}$ and restrict to the geodesics $\ell_{t} \subset \mathscr{C}_{t}$ corresponding to $\ell_{0}$. Fix a small $t>0$ and consider $p, q \in \partial N_{t}^{\delta}$ lying at distance $\delta$ from $\ell_{t}$ on opposite sides of $\ell_{t}$. The segment $[p, q]$ can be subdivided at its intersection point $x$ with $\ell_{t}$ into two subsegments to which Observation 4.3 applies, yielding

$$
\left\{\begin{array}{l}
d\left(f_{t}(p), f_{t}(x)\right) \leq(1-t) d(p, x)+L^{\prime} t \\
d\left(f_{t}(x), f_{t}(q)\right) \leq(1-t) d(x, q)+L^{\prime} t
\end{array}\right.
$$

Up to switching $p$ and $q$, we may assume that either $[p, x]$ projects to a pair of pants labeled 0 in $j_{t}\left(\Gamma_{g}\right) \backslash \mathbb{M}^{2} \simeq \Sigma_{g}$, or $[p, x]$ projects to a pair of pants labeled -1 and $[x, q]$ to a pair of pants labeled 1 .

Suppose that $[p, x]$ projects to a pair of pants labeled 0 in $j_{t}\left(\Gamma_{g}\right) \backslash \mathbb{T}^{2} \simeq \Sigma_{g}$. We first observe that, if $t$ is small enough (independently of $p$ ), then

$$
d\left(f_{t}(p), \ell_{0}\right) \geq \frac{3 \delta}{4} \text {. }
$$

Indeed, as in the proof of Observation 4.3, the inequality is true for $p \in \partial N_{t}^{\delta}$ in a fixed compact set $K^{\prime}$ independent of $t$, by condition (d) of Lemma 4.1 and (4-2), and we then use the fact that $f_{t}$ is $\left(j_{t}, j_{0}\right)$-equivariant under the stabilizer $S$ of $\ell_{0}$ in $\Gamma_{g}$, which acts cocompactly (by $j_{t}$ ) on the set of points at distance $\delta$ from $\ell_{t}$. By (4-5), if $t$ is small enough (independently of $p$ ), then the segment $\left[f_{t}(p), f_{t}(x)\right]$ spends at least $\delta / 4$ units of length in the complement $N_{0}^{\delta / 2}$ of the $\delta / 2$-neighborhood of $\mathscr{C}_{0}$. The point is that $\operatorname{Lip}_{y}(f)<1$ for all $y \in \mathbb{H}^{2} \backslash \mathscr{C}_{0}$ projecting to a pair of pants labeled 0 in $j_{0}\left(\Gamma_{g}\right) \backslash \mathbb{M}^{2} \simeq \Sigma_{g}$, and this bound is uniform in restriction to $N_{0}^{\delta / 2}$ since the function $p \mapsto \operatorname{Lip}_{p}(f)$ is upper semicontinuous and $j_{0}\left(\Gamma_{g}\right)$-invariant. Remark 2.1 thus implies the existence of a constant $\varepsilon>0$, independent of $t, \ell_{t}$, $p, x$, such that

$$
d\left(f \circ f_{t}(p), f \circ f_{t}(x)\right) \leq d\left(f_{t}(p), f_{t}(x)\right)-\varepsilon .
$$


Using the triangle inequality and the fact that $f$ is 1-Lipschitz, together with (4-4) and (4-6), we find

$$
\begin{aligned}
d\left(F_{t}(p), F_{t}(q)\right) & \leq d\left(f \circ f_{t}(p), f \circ f_{t}(x)\right)+d\left(f \circ f_{t}(x), f \circ f_{t}(q)\right) \\
& \leq(1-t) d(p, x)+L^{\prime} t-\varepsilon+(1-t) d(x, q)+L^{\prime} t,
\end{aligned}
$$

which is bounded by $(1-t) d(p, q)$ as soon as $t \leq \varepsilon /\left(2 L^{\prime}\right)$.

Suppose that $[p, x]$ projects to a pair of pants labeled -1 and $[x, q]$ to a pair of pants labeled 1. We then use the fact that the continuous map $f$ folds along $\ell_{0}=f_{t}\left(\ell_{t}\right)$. In restriction to the connected component of $\mathbb{H}^{2} \backslash \mathscr{C}_{0}$ containing $f_{t}(p)$ (resp. $f_{t}(q)$ ), it is an isometry preserving (resp. reversing) the orientation. In particular, $d\left(F_{t}(p), F_{t}(q)\right)<d\left(f_{t}(p), f_{t}(q)\right)$. Moreover, this inequality can be made uniform in the following sense: there exists $\varepsilon>0$ such that

$$
d\left(F_{t}(p), F_{t}(q)\right) \leq d\left(f_{t}(p), f_{t}(q)\right)-\varepsilon
$$

whenever $f_{t}(p)$ and $f_{t}(q)$ lie at distance at least $3 \delta / 4$ from $\ell_{0}$ (which is the case for $t$ small enough by (4-5)) and at distance at most $3 L^{\prime}$ from each other. By (4-4),

$$
d\left(f_{t}(p), f_{t}(q)\right) \leq(1-t) d(p, q)+2 L^{\prime} t,
$$

which implies

$$
d\left(F_{t}(p), F_{t}(q)\right) \leq(1-t) d(p, q)
$$

for $d(p, q) \leq 3 L^{\prime}$ as soon as $t \leq \varepsilon /\left(2 L^{\prime}\right)$ is small enough. If $d(p, q) \geq 3 L^{\prime}$, then applying the 1-Lipschitz map $f$ to (4-7) directly gives

$$
d\left(F_{t}(p), F_{t}(q)\right) \leq(1-t) d(p, q)+2 L^{\prime} t \leq\left(1-\frac{1}{3} t\right) d(p, q) .
$$

4D. Folding a given surface. We now prove the second statement of Theorem 1.1. Namely, given $\left[j_{0}\right] \in \operatorname{Rep}_{g}^{\mathrm{fd}}$ and an integer $k \in(-2 g+2,2 g-2)$, we construct $[\rho] \in \operatorname{Rep}_{g}^{\text {nfd }}$ with $\operatorname{eu}(\rho)=k$ that is strictly dominated by $\left[j_{0}\right]$.

It is easy to find $[\rho]$ with eu $(\rho)=k$ such that $\lambda_{\rho}(\gamma) \leq \lambda_{j_{0}}(\gamma)$ for all $\gamma \in \Gamma_{g}$ : just decompose $\Sigma_{g}$ into pairs of pants and assign arbitrary values $0,1,-1$ to each so that the sum is $k$. Consider a lamination $\Upsilon$ of $\Sigma_{g}$ consisting of all the cuffs together with a triskelion lamination inside each pair of pants labeled 0 , and let $c: \Sigma_{g} \backslash \Upsilon \rightarrow\{-1,1\}$ be a coloring taking the value -1 (resp. 1) on each pair of pants labeled -1 (resp. 1), and both values on each pair of pants labeled 0 . Folding along $\Upsilon$ with the coloring $c$ gives an element $[\rho] \in \operatorname{Rep}_{g}^{\text {nfd }}$ with $\lambda_{\rho}(\gamma) \leq \lambda_{j_{0}}(\gamma)$ for all $\gamma \in \Gamma_{g}$. However, we need a strict domination. The idea is to obtain $\rho$ by folding not $j_{0}$ but a small deformation of $j_{0}$. For this purpose, we use the following result, which is analogous to Lemma 4.1.

Lemma 4.4. Let $\Gamma$ be the fundamental group and $j_{0} \in \operatorname{Hom}(\Gamma, \operatorname{PSL}(2, \mathbb{R}))$ the holonomy of a compact, connected hyperbolic surface $\Sigma$ with nonempty geodesic 
boundary. Then there exist $t_{0}>0$ and a continuous family of representations $\left(j_{t}\right)_{0 \leq t \leq t_{0}}$ with the following properties:

(a) $\lambda_{j_{t}}(\gamma)=(1-t) \lambda_{j_{0}}(\gamma)$ for any $t \in\left[0, t_{0}\right]$ and any $\gamma \in \Gamma$ corresponding to a boundary component of $\Sigma$;

(b) $\sup _{\gamma \in \Gamma \backslash\{1\}} \lambda_{j_{t}}(\gamma) / \lambda_{j_{0}}(\gamma)<1$ for any $t \in\left(0, t_{0}\right]$;

(c) $j_{t}(\gamma)=j_{0}(\gamma)+O(t)$ for any $\gamma \in \Gamma$ as $t \rightarrow 0$, where both sides are seen as $2 \times 2$ real matrices with determinant 1 modulo $\pm \mathrm{Id}$;

(d) for any compact subset $K$ of $\mathbb{M}^{2}$ projecting to the interior of the convex core of $j_{0}(\Gamma) \backslash \mathbb{M}^{2}$, there exists $L>0$ such that

$$
d\left(p, f_{t}(p)\right) \leq L t
$$

for any $p \in K$, any $t \in\left[0, t_{0}\right]$, and any 1-Lipschitz, $\left(j_{0}, j_{t}\right)$-equivariant map $f_{t}: \mathbb{H}^{2} \rightarrow \mathbb{H}^{2}$.

As in the proof of Lemma 4.1, we construct the representations $j_{t}$ as holonomies

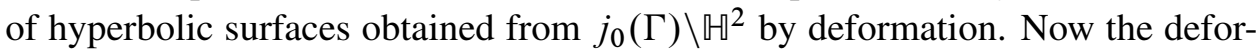
mation needs to be shortening instead of lengthening, so we use negative strip deformations.

Proof of Lemma 4.4. We view $\Sigma$ as the convex core of $j_{0}(\Gamma) \backslash \mathbb{M}{ }^{2}$. To shorten one boundary component $\beta$ of $\Sigma$, choose a finite collection of disjoint, biinfinite geodesic $\operatorname{arcs} \alpha_{1}, \ldots, \alpha_{n} \subset j_{0}(\Gamma) \backslash \mathbb{W}^{2}$, each crossing $\beta$ orthogonally twice, subdividing $\Sigma$ into right-angled hexagons and one-holed right-angled bigons. Near each $\alpha_{i}$, choose a second geodesic arc $\alpha_{i}^{\prime}$, also crossing $\beta$ twice, such that $\alpha_{i}, \alpha_{i}^{\prime}$ are closest at some points $p_{i}, p_{i}^{\prime} \in \Sigma$. We take all arcs to be pairwise disjoint. For every $i$, delete the hyperbolic strip $A_{i}$ bounded by $\alpha_{i}$ and $\alpha_{i}^{\prime}$ and glue the arcs back together isometrically, identifying $p_{i}$ with $p_{i}^{\prime}$. This yields a new complete hyperbolic surface with a compact convex core, equipped with a natural 1-Lipschitz map $\varsigma_{t}^{\beta}$ from $j_{0}(\Gamma) \backslash \mathbb{M}^{2}$ obtained by collapsing the strips $A_{i}$ to lines. The set $\varsigma_{t}^{\beta}(\Sigma)$ is strictly contained in the new convex core. The geodesic corresponding to $\beta$ is shorter in the new surface than in $\Sigma$. By adjusting the widths of the strips $A_{i}$, we may assume that the ratio of lengths is $1 /(1-t)$. Note that the appropriate widths for this ratio are in $O(t)$ as $t \rightarrow 0$. All lengths of geodesics corresponding to boundary components other than $\beta$ are unchanged.

Repeat the construction, iteratively, for all boundary components $\beta_{1}, \ldots, \beta_{r}$ of $\Sigma$, in some arbitrary order. We thus obtain a new complete hyperbolic surface $j_{t}(\Gamma) \backslash \mathbb{H}^{2}$, with a compact convex core $\Sigma_{t}$, such that $j_{t}$ satisfies (a). As in the proof of Lemma 4.1, up to replacing each $j_{t}$ with a conjugate under PSL $(2, \mathbb{R})$, we may assume that (c) is satisfied. To see that (b) and (d) also hold, we use the 
1-Lipschitz map $\varsigma_{t}:=\varsigma_{t}^{\beta_{r}} \circ \cdots \circ \varsigma_{t}^{\beta_{1}}$ from $\Sigma$ to $\Sigma_{t}$ and argue as in the proof of Lemma 4.1, switching $j_{t}$ and $j_{0}$.

As in Section $4 \mathrm{~B}$, we write $\Sigma_{g}=\Sigma^{1} \cup \cdots \cup \Sigma^{m}$, where $\Sigma^{i}$, for any $1 \leq i \leq m$, is a compact surface with boundary that is one of:

- a pair of pants labeled 0 ,

- a full connected component of the subsurface of $\Sigma_{g}$ made of pants labeled -1,

- or a full connected component of the subsurface of $\Sigma_{g}$ made of pants labeled 1.

Choose a small $\delta>0$ such that, in all hyperbolic metrics on $\Sigma_{g}$ which are close enough to that defined by $j_{0}$, any simple geodesic entering the $\delta$-neighborhood of the geodesic representative of a cuff of our chosen pants decomposition crosses the cuff. We use again the notation $\mathscr{C}_{0}, N_{0}^{\delta}, K$ from Section 4B. Applying Lemma 4.4 to $\Gamma^{i}:=\pi_{1}\left(\Sigma^{i}\right)$ and $j_{0}^{i}:=\left.j_{0}\right|_{\Gamma^{i}}$, we obtain continuous families of representations $\left(j_{t}^{i}\right)_{0 \leq t \leq t_{0}}$ for $1 \leq i \leq m$ satisfying (a)-(d), with a uniform constant $L>0$ for the compact set $K \subset \mathbb{H}^{2} \backslash \mathscr{C}_{0}$. For any $t \geq 0$, using (a), we can glue together the convex cores of the $j_{t}^{i}\left(\Gamma^{i}\right) \backslash \mathbb{M}^{2}$ following the same combinatorics as the $\Sigma^{i}$. This gives a closed hyperbolic surface of genus $g$, hence a holonomy representation $j_{t} \in \operatorname{Hom}\left(\Gamma_{g}, \operatorname{PSL}(2, \mathbb{R})\right)$. By (c), up to adjusting the twist parameters, we may assume that $j_{t}(\gamma)=j_{0}(\gamma)+O(t)$ for any $\gamma \in \Gamma_{g}$ as $t \rightarrow 0$, where both sides are seen as $2 \times 2$ real matrices with determinant 1 modulo \pm Id.

Recall the notation $\mathfrak{C}_{t}, N_{t}^{\delta}$ from Section 4B. By Proposition 3.9, there exist a family $\left(\rho_{t}\right)_{0 \leq t \leq t_{0}} \subset \operatorname{Hom}\left(\Gamma_{g}, \operatorname{PSL}(2, \mathbb{R})\right)$ of non-Fuchsian representations and, for any $t \in\left[0, t_{0}\right]$, a 1 -Lipschitz, $\left(j_{t}, \rho_{t}\right)$-equivariant map $\varphi_{t}: \mathbb{U}^{2} \rightarrow \mathbb{H}^{2}$ that is an orientation-preserving (resp. orientation-reversing) isometry in restriction to any connected subset of $\mathbb{H}^{2}$ projecting to a union of pants labeled -1 (resp. 1) in $j_{t}\left(\Gamma_{g}\right) \backslash \mathbb{T}^{2} \simeq \Sigma_{g}$, such that

$$
\operatorname{Lip}_{p}\left(\varphi_{t}\right) \leq C^{*}<1
$$

for all $t \in\left[0, t_{0}\right]$ and all $p \in N_{t}^{\delta}$ that project to a pair of pants labeled 0 in $j_{t}\left(\Gamma_{g}\right) \backslash \mathbb{M}^{2} \simeq \Sigma_{g}$, for some $C^{*}<1$ independent of $p$ and $t$.

We claim that, for $t>0$ small enough,

$$
\sup _{\gamma \in\left(\Gamma_{g}\right)_{s}} \frac{\lambda \rho_{t}(\gamma)}{\lambda j_{0}(\gamma)}<1
$$

which by Theorem 2.5 is enough to prove that $\left[\rho_{t}\right]$ is strictly dominated by $\left[j_{0}\right]$. Indeed, by (b) and Theorem 2.5, for any $1 \leq i \leq m$ and $t \in\left(0, t_{0}\right]$, there exists a $\left(\left.j_{t}\right|_{\Gamma^{i}},\left.j_{0}\right|_{\Gamma^{i}}\right)$-equivariant map $f_{t}^{i}: \mathbb{M}^{2} \rightarrow \mathbb{M}^{2}$ with $\operatorname{Lip}\left(f_{t}^{i}\right)<1$. Let $f_{t}$ be a $\left(j_{0}, j_{t}\right)$-equivariant map $\left(N_{0}^{\delta} \cup \mathscr{C}_{0}\right) \rightarrow \mathbb{M}^{2}$ such that:

- $f_{t}=f_{t}^{i}$ on the component of $K$ projecting to $\Sigma^{i}$ for all $1 \leq i \leq m$; 
- $f_{t}$ takes any geodesic line in $\mathscr{C}_{0}$ to the corresponding line in $\mathscr{C}_{t}$, multiplying all distances by the uniform factor $(1-t)$, and $d\left(x, f_{t}(x)\right) \leq L_{1} t$ for all $x \in \mathscr{C}_{0} \cap K$, for some $L_{1} \geq 0$ independent of $x$ and $t$.

Consider the $\left(j_{0}, \rho_{t}\right)$-equivariant map

$$
G_{t}:=\varphi_{t} \circ f_{t}:\left(N_{0}^{\delta} \cup \mathscr{C}_{0}\right) \longrightarrow \mathbb{M}^{2} .
$$

Any geodesic segment $I=[p, q]$ of $\mathbb{W}^{2}$ that projects to a closed geodesic of $j_{0}\left(\Gamma_{g}\right) \backslash \mathbb{M}^{2} \simeq \Sigma_{g}$ may be decomposed into subsegments $I_{1}, \ldots, I_{n}$ contained in $N_{0}^{\delta}$ alternating with subsegments $I_{1}^{\prime}, \ldots, I_{n}^{\prime}$ crossing connected components of $\mathbb{Q}^{2} \backslash N_{0}^{\delta}$. By contractivity of $f_{t}$, the map $G_{t}$ has Lipschitz constant strictly less than 1 on each connected component of $N_{0}^{\delta}$, hence moves the endpoints of each $I_{k}$ closer together by a uniform factor (independent of $I$ ). The subsegments $I_{k}^{\prime}$ are treated by the following lemma, which implies (4-9) and therefore completes the proof of the second statement of Theorem 1.1.

Lemma 4.5 (analogue of Lemma 4.2). For small enough $t>0$, there exists $C<1$ such that, for all $p, q \in \partial N_{0}^{\delta}$ lying at distance $\delta$ from a line $\ell_{0} \subset \mathscr{C}_{0}$ on opposite sides of $\ell_{0}$,

$$
d\left(G_{t}(p), G_{t}(q)\right) \leq C d(p, q) .
$$

The proof of Lemma 4.5 uses the following observation, which is identical to Observation 4.3 after exchanging $j_{0}$ and $j_{t}$.

Observation 4.6. There exists $L^{\prime} \geq 0$ such that, for any small enough $t>0$, any $p \in \partial N_{0}^{\delta}$ at distance $\delta$ from a geodesic $\ell_{0} \subset \mathscr{C}_{0}$, and any $x \in \ell_{0}$,

$$
d\left(f_{t}(p), f_{t}(x)\right) \leq(1-t) d(p, x)+L^{\prime} t .
$$

Proof of Lemma 4.5. We argue as in the proof of Lemma 4.2, but switch $j_{0}$ and $j_{t}$ and use (4-8) to obtain the analogue

$$
d\left(\varphi_{t} \circ f_{t}(p), \varphi_{t} \circ f_{t}(x)\right) \leq d\left(f_{t}(p), f_{t}(x)\right)-\varepsilon
$$

of (4-6) when $[p, x]$ projects to a pair of pants labeled 0 in $j_{0}\left(\Gamma_{g}\right) \backslash \mathbb{M}^{2} \simeq \Sigma_{g}$.

\section{Acknowledgements}

We would like to thank Bertrand Deroin and Nicolas Tholozan for sharing their thoughts on the subject with us. The third author is grateful to Antonin Guilloux, Julien Marché, and Richard Wentworth for their support and for helpful conversations. 


\section{References}

[Bonahon 1996] F. Bonahon, "Shearing hyperbolic surfaces, bending pleated surfaces and Thurston's symplectic form", Ann. Fac. Sci. Toulouse Math. (6) 5:2 (1996), 233-297. MR 97i:57011 ZBL 0880.57005

[Bridson and Haefliger 1999] M. R. Bridson and A. Haefliger, Metric spaces of non-positive curvature, Grundlehren der Mathematischen Wissenschaften 319, Springer, Berlin, 1999. MR 2000k:53038 Zbl 0988.53001

[Calegari 2004] D. Calegari, "Circular groups, planar groups, and the Euler class", pp. 431-491 in Proceedings of the Casson Fest (Fayetteville, AR/Austin, TX, 2003), edited by C. Gordon and Y. Rieck, Geometry and Topology Monographs 7, Mathematical Sciences Publishers, Coventry, 2004. MR 2006i:57038 Zbl 1181.57022

[Danciger et al. 2014] J. Danciger, F. Guéritaud, and F. Kassel, "Margulis spacetimes via the arc complex”, preprint, 2014. arXiv 1407.5422

[Deroin and Tholozan 2013] B. Deroin and N. Tholozan, "Dominating surface group representations by Fuchsian ones", preprint, 2013. arXiv 1311.2919

[Gallo et al. 2000] D. Gallo, M. Kapovich, and A. Marden, "The monodromy groups of Schwarzian equations on closed Riemann surfaces", Ann. of Math. (2) 151:2 (2000), 625-704. MR 2002j:57029 Zbl 0977.30028

[Ghys 2001] É. Ghys, "Groups acting on the circle", Enseign. Math. (2) 47:3-4 (2001), 329-407. MR 2003a:37032 Zbl 1044.37033

[Goldman 1985] W. M. Goldman, "Nonstandard Lorentz space forms", J. Differential Geom. 21:2 (1985), 301-308. MR 87h:53093 Zbl 0591.53051

[Goldman 1987] W. M. Goldman, "Projective structures with Fuchsian holonomy", J. Differential Geom. 25:3 (1987), 297-326. MR 88i:57006 Zbl 0595.57012

[Goldman 1988] W. M. Goldman, "Topological components of spaces of representations", Invent. Math. 93:3 (1988), 557-607. MR 89m:57001 Zbl 0655.57019

[Goldman and Xia 2011] W. M. Goldman and E. Z. Xia, "Ergodicity of mapping class group actions on SU(2)-character varieties", pp. 591-608 in Geometry, rigidity, and group actions (Chicago, 2007), edited by B. Farb and D. Fisher, University of Chicago Press, 2011. MR 2012k:22028 Zbl 1283.22016 arXiv 0901.1402

[Guéritaud and Kassel 2013] F. Guéritaud and F. Kassel, "Maximally stretched laminations on geometrically finite hyperbolic manifolds", preprint, 2013. arXiv 1307.0250

[Guéritaud et al. 2015] F. Guéritaud, O. Guichard, F. Kassel, and A. Wienhard, "Anosov representations and proper actions", preprint, 2015. To appear in Geom. Topol. arXiv 1502.03811

[Hitchin 1987] N. J. Hitchin, "The self-duality equations on a Riemann surface", Proc. London Math. Soc. (3) 55:1 (1987), 59-126. MR 89a:32021 Zbl 0634.53045

[Kassel 2008] F. Kassel, "Proper actions on corank-one reductive homogeneous spaces", J. Lie Theory 18:4 (2008), 961-978. MR 2010g:22019 Zbl 1173.22009

[Kassel 2009] F. Kassel, Quotients compacts d'espaces homogènes réels ou p-adiques, Ph.D. thesis, Université Paris-Sud 11, 2009, Available at http://math.univ-lille1.fr/ kassel/These.pdf.

[Klingler 1996] B. Klingler, "Complétude des variétés Lorentziennes à courbure constante", Math. Ann. 306:2 (1996), 353-370. MR 97g:53082 Zbl 0862.53048

[Kobayashi 1998] T. Kobayashi, "Deformation of compact Clifford-Klein forms of indefiniteRiemannian homogeneous manifolds", Math. Ann. 310:3 (1998), 395-409. MR 99b:53074 ZBL 0891.22014 
[Kulkarni and Raymond 1985] R. S. Kulkarni and F. Raymond, "3-dimensional Lorentz spaceforms and Seifert fiber spaces”, J. Differential Geom. 21:2 (1985), 231-268. MR 87h:53092 Zbl 0563.57004

[Papadopoulos and Théret 2010] A. Papadopoulos and G. Théret, "Shortening all the simple closed geodesics on surfaces with boundary”, Proc. Amer. Math. Soc. 138:5 (2010), 1775-1784. MR 2011d: 30111 Zbl 1197.32006

[Salein 1999] F. Salein, Variétés anti-de Sitter de dimension 3, Ph.D. thesis, École Normale Supérieure de Lyon, 1999, Available at http://www.umpa.ens-lyon.fr/ zeghib/these.salein.pdf.

[Salein 2000] F. Salein, "Variétés anti-de Sitter de dimension 3 exotiques", Ann. Inst. Fourier (Grenoble) 50:1 (2000), 257-284. MR 2001h:57020 Zbl 0951.53047

[Selberg 1960] A. Selberg, "On discontinuous groups in higher-dimensional symmetric spaces", pp. 147-164 in Contributions to function theory (Bombay, 1960), Tata Institute of Fundamental Research, Bombay, 1960. Reprinted in Collected papers, A. Selberg, Vol. 1, pp. 475-492, Springer, Berlin, 1989. MR 24 \#A188 Zbl 0201.36603

[Thurston 1980] W. P. Thurston, "The geometry and topology of three-manifolds", 1980, Available at http://library.msri.org/books/gt3m. Lecture notes.

[Thurston 1986] W. P. Thurston, "Minimal stretch maps between hyperbolic surfaces", preprint, 1986. arXiv math/9801039

[Wolff 2011] M. Wolff, "Connected components of the compactification of representation spaces of surface groups”, Geom. Topol. 15:3 (2011), 1225-1295. MR 2825313 Zbl 1226.57027

Received November 16, 2013. Revised July 7, 2014.

FRANÇOIS GUÉRITAUD

CNRS AND UNIVERSITÉ LILLE 1

Laboratoire Paul Painlevé, Université Lille 1

59655 Villeneuve d’ AsCQ CEDEX

FRANCE

francois.gueritaud@math.univ-lille1.fr

FANNY KASSEL

CNRS AND UNIVERSITÉ LILLE 1

Laboratoire Paul Painlevé, Université Lille 1

59655 Villeneuve d' AscQ CEdeX

FRANCE

fanny.kassel@math.univ-lille1.fr

MAXIME WOLFF

Institut de Mathématiques de Jussieu-Paris Rive Gauche, CNRS

Université Pierre et MARIE CURIE - PARis 6

4 PLACE JUSSIEU - CASE 247

75005 PARIS 05

FRANCE

maxime.wolff@imj-prg.fr 


\title{
PACIFIC JOURNAL OF MATHEMATICS
}

\author{
msp.org/pjm
}

Founded in 1951 by E. F. Beckenbach (1906-1982) and F. Wolf (1904-1989)

\section{EDITORS}

Don Blasius (Managing Editor)

Department of Mathematics

University of California

Los Angeles, CA 90095-1555

blasius@math.ucla.edu

\author{
Paul Balmer \\ Department of Mathematics \\ University of California \\ Los Angeles, CA 90095-1555 \\ balmer@math.ucla.edu \\ Robert Finn \\ Department of Mathematics \\ Stanford University \\ Stanford, CA 94305-2125 \\ finn@math.stanford.edu \\ Sorin Popa \\ Department of Mathematics \\ University of California \\ Los Angeles, CA 90095-1555 \\ popa@math.ucla.edu
}

\author{
Vyjayanthi Chari \\ Department of Mathematics \\ University of California \\ Riverside, CA 92521-0135 \\ chari@math.ucr.edu \\ Kefeng Liu \\ Department of Mathematics \\ University of California \\ Los Angeles, CA 90095-1555 \\ liu@math.ucla.edu \\ Jie Qing \\ Department of Mathematics \\ University of California \\ Santa Cruz, CA 95064 \\ qing@ cats.ucsc.edu
}

\section{PRODUCTION}

Silvio Levy, Scientific Editor, production@msp.org

\section{SUPPORTING INSTITUTIONS}

ACADEMIA SINICA, TAIPEI

CALIFORNIA INST. OF TECHNOLOGY

INST. DE MATEMÁTICA PURA E APLICADA

KEIO UNIVERSITY

MATH. SCIENCES RESEARCH INSTITUTE

NEW MEXICO STATE UNIV.

OREGON STATE UNIV.

\author{
STANFORD UNIVERSITY \\ UNIV. OF BRITISH COLUMBIA \\ UNIV. OF CALIFORNIA, BERKELEY \\ UNIV. OF CALIFORNIA, DAVIS \\ UNIV. OF CALIFORNIA, LOS ANGELES \\ UNIV. OF CALIFORNIA, RIVERSIDE \\ UNIV. OF CALIFORNIA, SAN DIEGO \\ UNIV. OF CALIF., SANTA BARBARA
}

\author{
Daryl Cooper \\ Department of Mathematics \\ University of California \\ Santa Barbara, CA 93106-3080 \\ cooper@math.ucsb.edu \\ Jiang-Hua Lu \\ Department of Mathematics \\ The University of Hong Kong \\ Pokfulam Rd., Hong Kong \\ jhlu@maths.hku.hk \\ Paul Yang \\ Department of Mathematics \\ Princeton University \\ Princeton NJ 08544-1000 \\ yang@math.princeton.edu
}

These supporting institutions contribute to the cost of publication of this Journal, but they are not owners or publishers and have no responsibility for its contents or policies.

See inside back cover or msp.org/pjm for submission instructions.

The subscription price for 2015 is US \$420/year for the electronic version, and \$570/year for print and electronic.

Subscriptions, requests for back issues and changes of subscribers address should be sent to Pacific Journal of Mathematics, P.O. Box 4163, Berkeley, CA 94704-0163, U.S.A. The Pacific Journal of Mathematics is indexed by Mathematical Reviews, Zentralblatt MATH, PASCAL CNRS Index, Referativnyi Zhurnal, Current Mathematical Publications and Web of Knowledge (Science Citation Index).

The Pacific Journal of Mathematics (ISSN 0030-8730) at the University of California, c/o Department of Mathematics, 798 Evans Hall \#3840, Berkeley, CA 94720-3840, is published twelve times a year. Periodical rate postage paid at Berkeley, CA 94704, and additional mailing offices. POSTMASTER: send address changes to Pacific Journal of Mathematics, P.O. Box 4163, Berkeley, CA 94704-0163.

PJM peer review and production are managed by EditFLOW ${ }^{\circledR}$ from Mathematical Sciences Publishers.

\section{PUBLISHED BY}

\section{mathematical sciences publishers \\ nonprofit scientific publishing}

http://msp.org/

(C) 2015 Mathematical Sciences Publishers 


\section{PACIFIC JOURNAL OF MATHEMATICS}

Volume $275 \quad$ No. $2 \quad$ June 2015

A combinatorial characterization of tight fusion frames

MARCIN BOWNIK, KURT LUOTO and EDWARD RICHMOND

Combinatorics of finite abelian groups and Weil representations

295

KUnAL DUTTA and AMritANSHU PRASAD

Compact anti-de Sitter 3-manifolds and folded hyperbolic structures on

325 surfaces

FRANÇOIS GUÉRITAUd, FANNY KASSEL and MAXIME WOLFF

Circular handle decompositions of free genus one knots

361

FABIOLA MANJARREZ-GUTIÉRREZ, VÍCTOR NÚÑEZ and

ENRIQUE RAMÍREZ-LOSADA

A pointwise a-priori estimate for the $\bar{\partial}$-Neumann problem on weakly 409 pseudoconvex domains

R. MichaEL RANGE

Explicit Hilbert-Kunz functions of $2 \times 2$ determinantal rings

MARCUS ROBINSON and IRENA SWANSON

The Johnson-Morita theory for the ring of Fricke characters of free groups

\section{TAKAO SATOH}

Global representations of the conformal group and eigenspaces of the Yamabe operator on $S^{1} \times S^{n}$

MARK R. SEPANSKI and Jose A. Franco

Rota-Baxter operators on the polynomial algebra, integration, and averaging operators

Shanghua Zheng, Li GuO and MARKus Rosenkranz

Correction to the article Quiver grassmannians, quiver varieties and the preprojective algebra

Alistair Savage and Peter Tingley 